\title{
Hierarchical assembly pathways of spermine induced tubulin conical-spiral architectures
}

\author{
Raviv Dharan ${ }^{1,2}$, Asaf Shemesh ${ }^{1,3}$, Abigail Millgram ${ }^{1,3}$, Yael Levi-Kalisman ${ }^{3,4}$, Israel \\ Ringel $^{2 *}$ and Uri Ravivit ${ }^{1,3}$ \\ ${ }^{1}$ Institute of Chemistry, ${ }^{2}$ Institute for Drug Research, ${ }^{3}$ The Center for Nanoscience and \\ Nanotechnology, ${ }^{4}$ Institute of life sciences, \\ The Hebrew University of Jerusalem, Jerusalem, Israel \\ *Corresponding authors: uri.raviv@mail.huji.ac.il, israelr@ekmd.huji.ac.il
}

Tubulin, an essential cytoskeletal protein, assembles into various morphologies by interacting with cellular factors. Spermine, an endogenous polyamine, promotes and stabilizes tubulin assemblies. Yet, the assembled structures and their formation pathways are poorly known. Here we show that spermine induced tubulin to assemble in vitro into hierarchical architectures, based on a tubulin conical-spiral (TCS) subunit. Using solution X-ray scattering and cryo-TEM, we showed that with progressive increase of spermine concentration, tubulin-dimers assembled into a tubulin helical-pitch (or a short TCS), TCSs, TCS that stacked into tubes through base-to-top packing, antiparallel bundles of TCS tubes in a quasi-hexagonal symmetry, and eventually twisted hexagonal bundles of inverted tubulin tubules. Time-resolved experiments revealed that tubulin assemblies formed at low spermine concentrations were precursors of the assemblies formed at higher spermine concentrations. The results provide insight into the variety of morphologies that tubulin can form, and contribute to our understanding of the fundamental interactions that control the composition and construction of protein-based biomaterials. 
Microtubules (MTs) are filaments made of polymerized $\alpha \beta$ tubulin dimers, essential for cellular activities including cell division, morphogenesis, and intracellular trafficking. The equilibrium between dimers and MT polymers is essential for their versatile biological functions and can be controlled by nucleotides, ions, proteins, or small molecules ${ }^{1,2}$. Depending on solution composition and temperature, tubulin can be assembled, aside from MTs, into rings, sheets, or other tubulin assemblies ${ }^{3,4,5}$. The large number of structures, assembled from the basic tubulin dimer, points to a flexible subunit, bearing several binding sites at multiple locations, enabling dimer-dimer binding and interactions with various factors. For example, hydrolysis of GTP-tubulin for GDP-tubulin, decreases the rate of exchange between straight and curved tubulin conformations $^{6}$. GTP-tubulin promotes the assembly of MTs ${ }^{7}$, whereas GDP-tubulin triggers their disassembly and promotes the assembly of tubulin-rings ${ }^{3,8}$. The intrinsic dynamics of tubulin is needed for constructing cellular structures such as the mitotic spindle, cilia, or axons ${ }^{9,10,11}$.

Tubulin is highly negatively charged. Much of its charge is concentrated in the Cterminal tail ${ }^{12}$, whereas the other negative sites are distributed along the dimer having varying cations binding affinities ${ }^{13}$. The electrostatic repulsion between the dimers impede polymerization ${ }^{14}, 15$. Relatively high concentrations of tubulin dimers are therefore required to overcome their spontaneous self-assembly barrier. The negative charge can be reduced by chemical modifications such as C-terminal cleavage ${ }^{16}$ or by positively charged agents such as MT-associated proteins (MAPs) or polycations ${ }^{17,}{ }^{18}$. Natural polyamines are cellular polycations, present at fluctuating concentrations, reaching about $1 \mathrm{mM}$ at certain cell cycle stages $^{19,20,21}$, and interact with anionic cytoskeletal filaments such as actin and $\mathrm{MTs}^{22}$. Among them, tetravalent spermine was found to be effective in promoting tubulin assembly and bundle formation ${ }^{23,24}$, most 
likely by forming ion-bridges ${ }^{25}, 26$. Here we show the effect of spermine, at millimolar concentrations, on tubulin assembly at steady state. We then followed in real-time the multiple phase transitions of the assemblies into hierarchical architectures, including macro-structures that are based on a tubulin conical-spiral (TCS) subunit, discovered in this study (Fig. 1). The rich phases discovered here extend the opportunities to design supramolecular biomaterials ${ }^{27}$. 

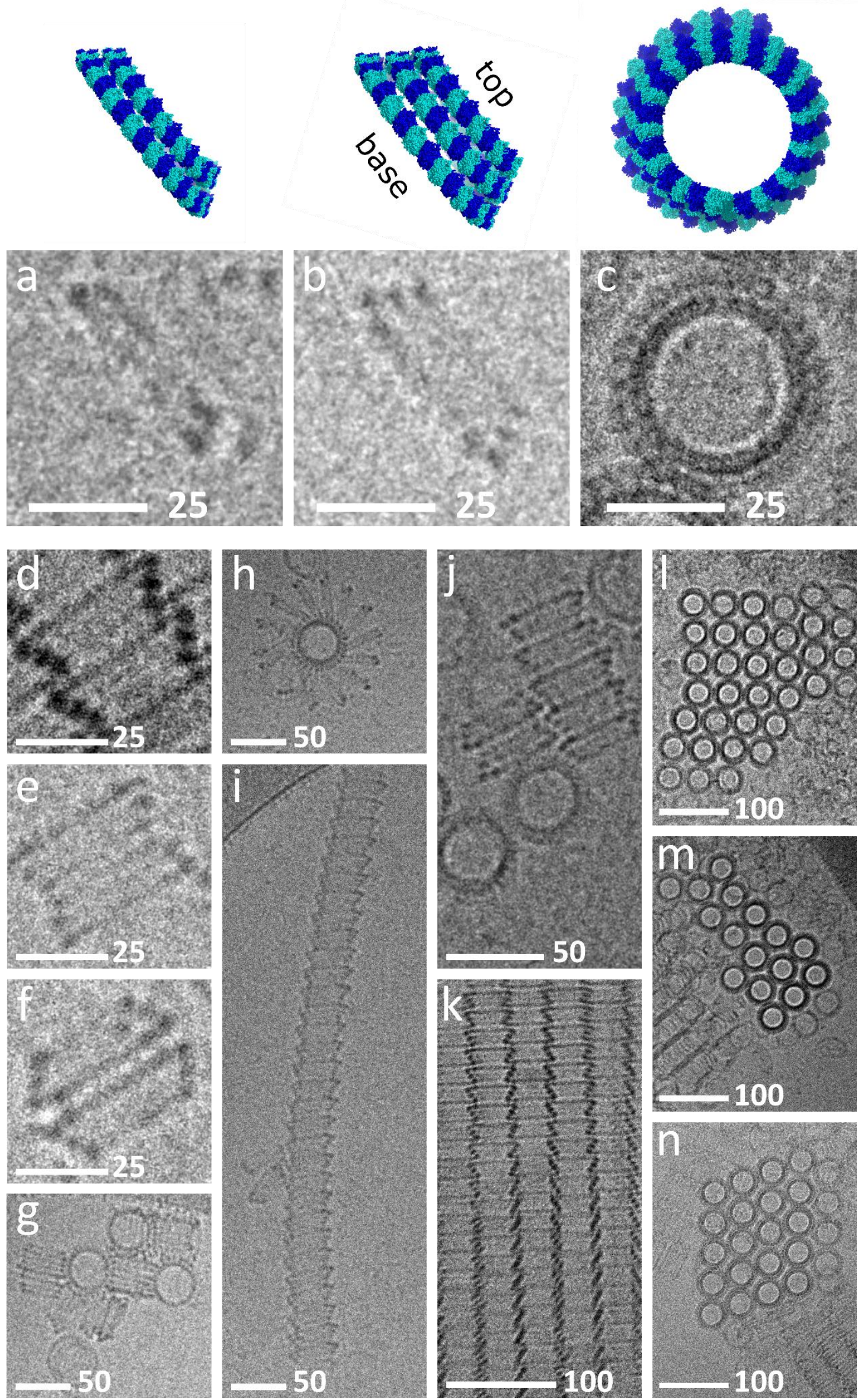
Figure 1| Cryo-TEM images of tubulin conical-spiral (TCS) and its hierarchical assemblies. a-c. Side (a, b) and top (c) views of a helical protofilament forming between 1.5 (a) and 3-pitch (b, c) TCS subunits. Schematic illustrations of the TCS subunits are shown above the images. $\mathbf{d - h}$. TCS subunits assembled vertically: base-totop (d), top-to-top (e), base-to-base (f), or perpendicularly to the central tubular axis (g, h). i. a TCS-tube (TCST) with extended base-to-top interactions. j-n. Highly ordered TCSTs, associated in an antiparallel alignment $(\mathbf{j}, \mathbf{k})$ into bundles of 2D-cubic (l) and 2D quasi-hexagonal lattices $(\mathbf{m}, \mathbf{n})$. In all experiments, $4 \mathrm{mg} / \mathrm{ml}$ (except in i: $2 \mathrm{mg} / \mathrm{ml}$ ) tubulin were incubated at $9{ }^{\circ} \mathrm{C}$ for $48 \mathrm{~h}$ with $5 \mathrm{mM}$ spermine. Scale bars are in nm units.

\section{$\underline{\text { Formation of tubulin conical-spiral and its hierarchical assemblies }}$}

Tubulin, purified from porcine brain ${ }^{28}$, was mixed with spermine tetrahydrochloride in a PEM50 buffer to a final concentration of $4 \mathrm{mg} / \mathrm{ml}$ tubulin and the desired spermine concentration (see Methods). Cryo-TEM images revealed a remarkable ensemble of TCS-based assemblies (Fig. 1). The structures were, most likely, constructed from protofilaments (PFs) that adopted helical conformations with gradually changing diameters, forming conical-spiral like-structures. The TCS subunits usually included between 1.5 and 3 helical pitches, which on a side or top view had between two (Fig. 1a, c) and three (Fig. 1b) diameters. Each TCS subunit had a base and a top, and could interact vertically or laterally with other subunits. Vertical interactions included baseto-top (Fig 1d), top-to-top (Fig. 1e), and base-to-base (Fig. 1f) TCS associations. In the lateral interactions, TCSs assembled into short tubules, attached to each other by positioning their tubular axes vertically (Fig. 1g), or individual TCS subunits surrounded another subunit (Fig. 1h). It is possible that in the latter mode of association, TCSs interacted with coexisting tubulin helical-pitch (THP) (or short TCS) structures 
(see below). The dominating population, however, contained TCS-tubes (TCSTs) of varying lengths. In the long tubes, TCS usually contained three helical pitches with base-to-top being the most abundant form of association (Fig. 1i). Remarkably, TCSTs further assembled into tight bundles of antiparallel TCSTs (Fig. 1j, k) with 2D-cubic or 2D-quasi hexagonal symmetries (Fig. 11-n). We note that the top view images (Fig. 1c, 1-n) show relatively short ( 200 nm or less) TCSTs because longer structures are limited by the ice film thickness. It is likely that thermal fluctuations assisted the gradual transition from cubic to the more compact and possibly more stable quasi-hexagonal lattice. In the quasi-hexagonal lattice, each tube had four closely packed antiparallel neighbors and two slightly separated parallel neighbors.

From THP to TCS to inverted tubulin tubules - phase transitions and assembly pathways

The effect of spermine concentration on tubulin assembly was studied by small angle X-ray scattering (SAXS). Figure 2a shows synchrotron SAXS measurements of 4 $\mathrm{mg} / \mathrm{ml}$ tubulin incubated at $9{ }^{\circ} \mathrm{C}$ for $48 \mathrm{~h}$ with $1-20 \mathrm{mM}$ spermine. At $1 \mathrm{mM}$ spermine, only THPs formed (Fig. 2a and Extended Data Figs. 1a, 2a, b). There is a difference between the SAXS signal of THPs and that of tubulin-single-rings ${ }^{28}$, attributed to the higher number of dimers in the THP structure and its larger (by about $2 \mathrm{~nm}$ ) average diameter (Extended Data Fig. 1d). It is likely that THP has also assumed a short TCS structure, as it evolved into TCS at higher spermine concentrations. Between 1.5 and 5 $\mathrm{mM}$ spermine, THPs coexisted with the TCS-based structures (Fig. 2b and Extended Data Fig. 3a); the mass fraction of THPs gradually decreased while TCSTs increased with elevated spermine concentration (Fig. 2c). Between 7.5 and $12.5 \mathrm{mM}$ TCS-based structures were observed (Fig. 2a, c, d, and Extended Data Figs. 2g-j, 3b) in coexistence 
with inverted tubulin tubes (ITTs). ITTs are twisted hexagonal bundles of 1-start helices, constructed from curved tubulin PFs, in which the outer tubulin surface corresponds to the inner surface of $\mathrm{MTs}^{24}$. The base-to-base and top-to-top associations in TCS assemblies were still observed at $10 \mathrm{mM}$ spermine (Extended Data Fig. 2g-j), suggesting that those modes of assembly were transition states between TCSTs and ITTs. When tubulin was incubated with 15 to $30 \mathrm{mM}$ spermine, only highly ordered twisted hexagonal bundles of ITTs formed (Fig. 2a and Extended Data Fig. 2k, 1). At higher spermine concentrations, the chemical potential of spermine in the solution was high, hence an excessive fraction of spermine adsorbed onto the tubulin and created a positively charged surface. The repulsive electrostatic interactions between the positively charged surfaces led to resolubilization of the tubulin assemblies (Extended Data Fig. 1c $)^{29,30}$. Our data show that increasing spermine concentration induced a complex phase behavior of tubulin assembly into THP, TCS and two distinct arrays of macroion complexes (bundles of TCSTs and ITTs) ${ }^{31}$. 
a

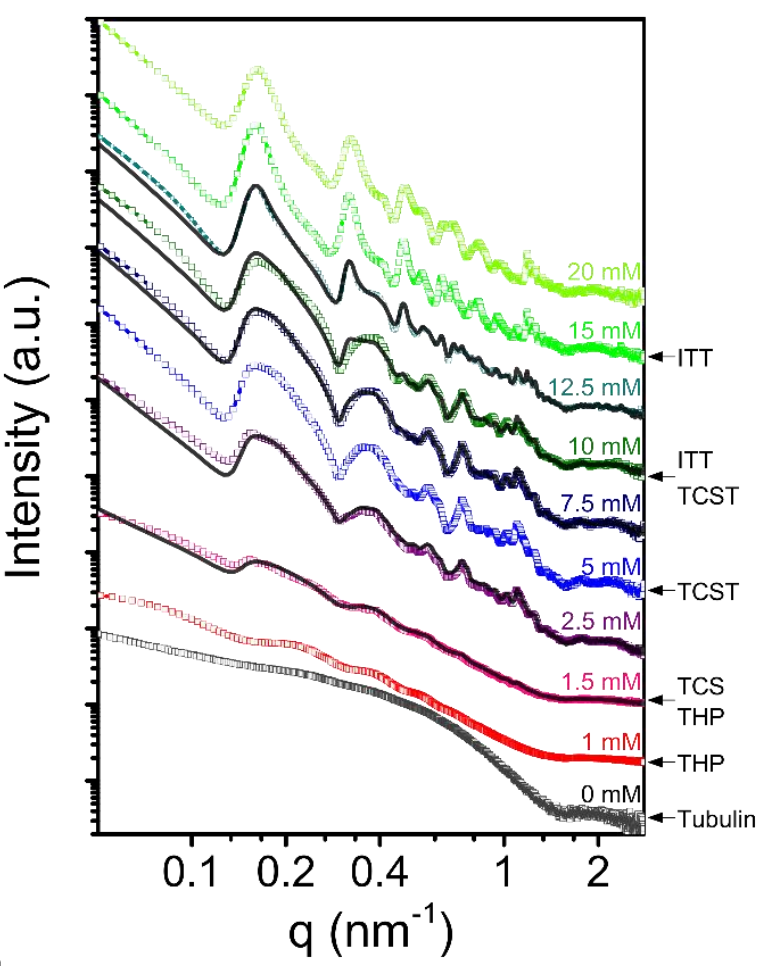

b

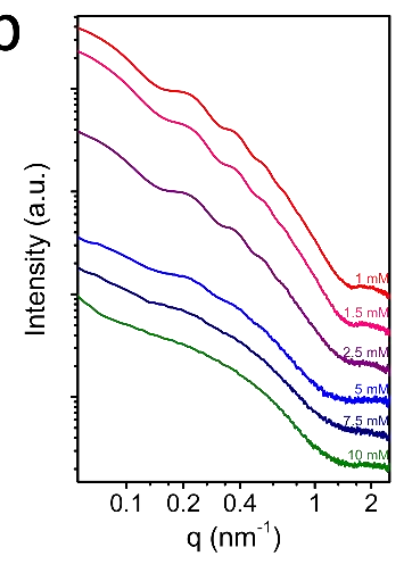

$\mathrm{C}$

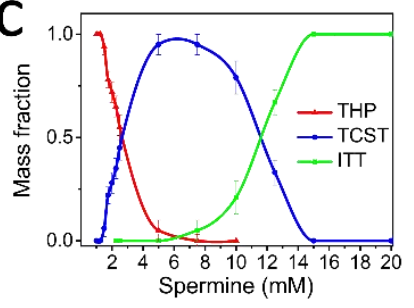

d
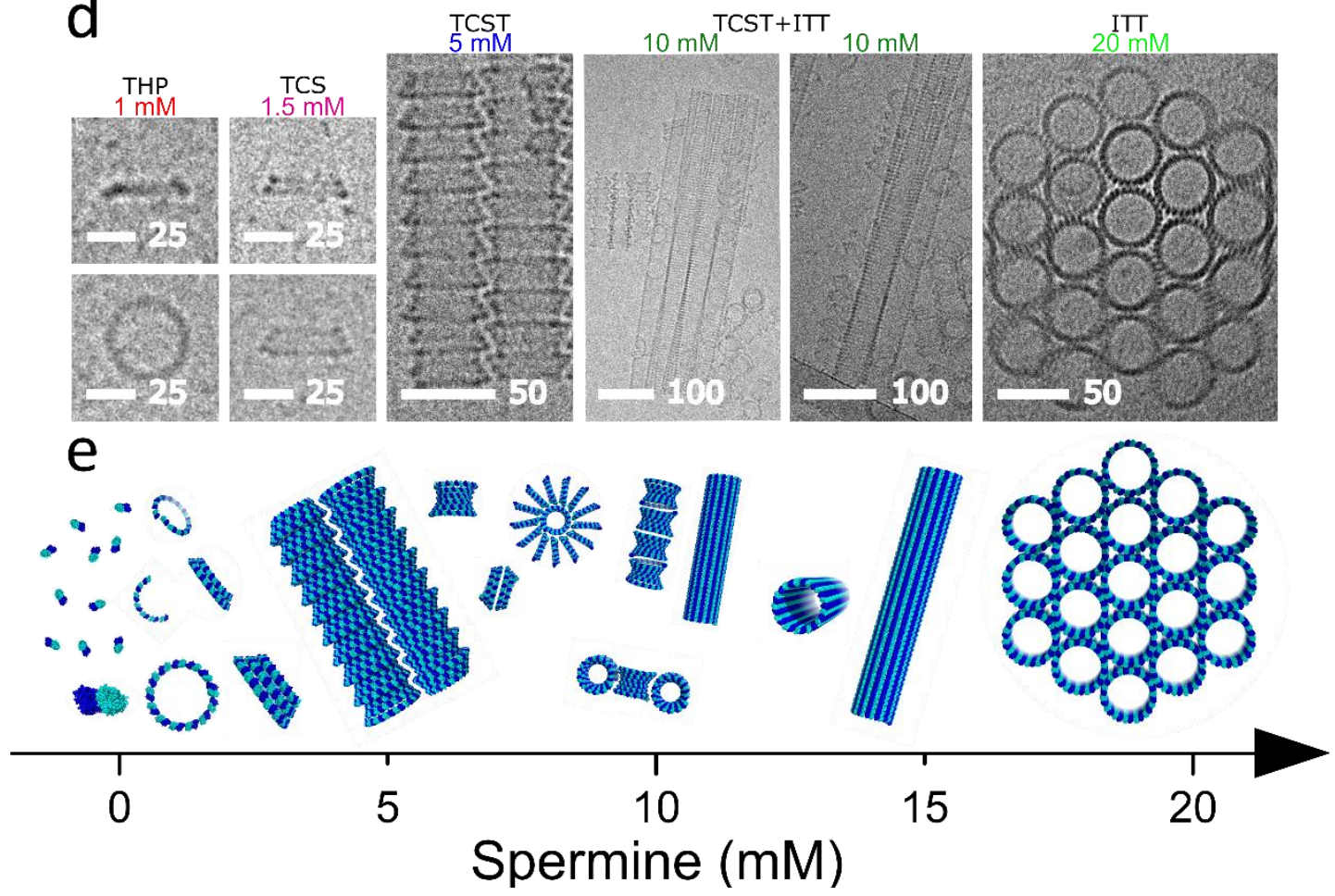

Figure 2| Spermine-induced phase transitions. a. Azimuthally-averaged buffersubtracted (0-2.5 $\mathrm{mM}$ spermine) or supernatant-subtracted $(5-20 \mathrm{mM}$ spermine $)$ synchrotron SAXS curves (open square symbol) depicting the phase transitions. Black curves are linear combinations of the SAXS curves obtained with 1, 5 and $15 \mathrm{mM}$ spermine. b. Azimuthally-averaged buffer-subtracted synchrotron SAXS curves from 
the supernatants of $4 \mathrm{mg} / \mathrm{ml}$ tubulin incubated with $1-10 \mathrm{mM}$ spermine, showing the coexisting free tubulin dimers and THP structures, contributing oscillations, similar to tubulin ring structures ${ }^{28}$. c. Mass fractions of THP, TCST and ITT assemblies as a function of spermine concentration, based on the data analysis shown in a and Extended data Fig. 3a-b. d. Cryo-TEM images of tubulin assemblies in the presence of the indicated spermine concentration. Scale bars are in $\mathrm{nm}$ units. e. Schematic illustration of the coexisting tubulin assemblies as a function of spermine concentration (tubulin dimers, curved protofilaments, THPs, TCSs, antiparallel TCSTs, base-to-base and topto-top TCS tubules and ITTs). Cartoons are not to scale. In all experiments, $4 \mathrm{mg} / \mathrm{ml}$ tubulin were incubated with the relevant spermine concentration for $48 \mathrm{~h}$ at $9{ }^{\circ} \mathrm{C}$ (apart from the case of no added spermine in $\mathbf{a}$ ).

The assembly pathways of THPs, TCSs, TCSTs and ITTs were examined using timeresolved synchrotron SAXS and time-resolved cryo-TEM measurements at $9{ }^{\circ} \mathrm{C}$ (Fig. 3a-c and Extended Data Figs. 4-6). When $4 \mathrm{mg} / \mathrm{ml}$ tubulin were mixed with $1 \mathrm{mM}$ spermine, the assembly initiated from tubulin dimers and small tubulin oligomers within less than $50 \mathrm{msec}$, THPs formed within $1 \mathrm{sec}$ (Fig. 3a), and remained stable for at least $48 \mathrm{~h}$. When tubulin was mixed with $5 \mathrm{mM}$ spermine (Fig. 3b), THPs formed even faster (within $0.22 \mathrm{sec}$, Extended Data Fig. 4d) and rapidly evolved (within a second or less) into TCS subunits. We attribute the fast increase of the intensity at $q=0.17$ (corresponding to a characteristic correlation distance of about $43 \mathrm{~nm}$ ) to association of TCS subunits into larger assemblies in various configurations, as observed in our cryo-TEM images (Fig. 3b and Extended Data Fig. 5). These configurations gradually transformed into the antiparallel TCSTs and the several coexisting hierarchical TCS structures that remained stable for days. 
Interestingly, the same sequence of assembly-intermediates was observed for $20 \mathrm{mM}$ spermine reaction (Fig. 3c). The assembly of THPs and TCS subunits, however, was slower compared with the $5 \mathrm{mM}$ reaction (Extended Data Fig. 4c, d), possibly owing to kinetic barriers. At $20 \mathrm{mM}$, TCS subunits immediately associated with each other and formed intermediates, similar to those in the $5 \mathrm{mM}$ reaction (Fig. $3 \mathrm{c}$ and Extended Data Figs. 4c, d, 6). At $20 \mathrm{mM}$ spermine, however, within 48 h, all TCS-based hierarchical structures completely transformed into the well-ordered twisted ITT hexagonal bundles.

Notably, the time-resolved results revealed that tubulin assemblies formed at low spermine concentrations were precursors of assemblies (TCST and ITT bundles) formed at higher spermine concentration (Fig. 3e).

To get additional insight into the transformation of TCS assemblies into ITT, we prepared a TCST-rich sample: $4 \mathrm{mg} / \mathrm{ml}$ tubulin incubated for $48 \mathrm{~h}$ at $9{ }^{\circ} \mathrm{C}$ with $5 \mathrm{mM}$ spermine. The solution was then exposed to $30 \mathrm{mM}$ spermine for additional $48 \mathrm{~h}$ and measured by synchrotron SAXS and cryo-TEM as a function of time (Fig. 3d, Extended Data Figs. 3c, 7). At $9{ }^{\circ} \mathrm{C}$, TCST bundles remained kinetically trapped. At $25{ }^{\circ} \mathrm{C}$, however, most of TCSTs population transformed into ITTs. Fig. 3d shows that TCST bundles initially disassembled into short TCSTs and TCS subunits, which then gradually transformed into ITTs (consistent with the direct assembly pathway of ITTs, Fig. 3e). Raising only the temperature to $25^{\circ} \mathrm{C}$ for $48 \mathrm{~h}$, without adding spermine, did not lead to any change (Fig. 3d, black broken curve). The polydispersity of the sample containing ITTs assembled from bundles of TCSTs was higher than ITTs assembled directly from tubulin dimers (based on the shallower minima and maxima in the scattering curves and the top view images in Fig. 3d and Extended Data Fig. 7). Similar polydispersity was reported for ITTs that formed from bundles of $\mathrm{MTs}^{24}$. 
a

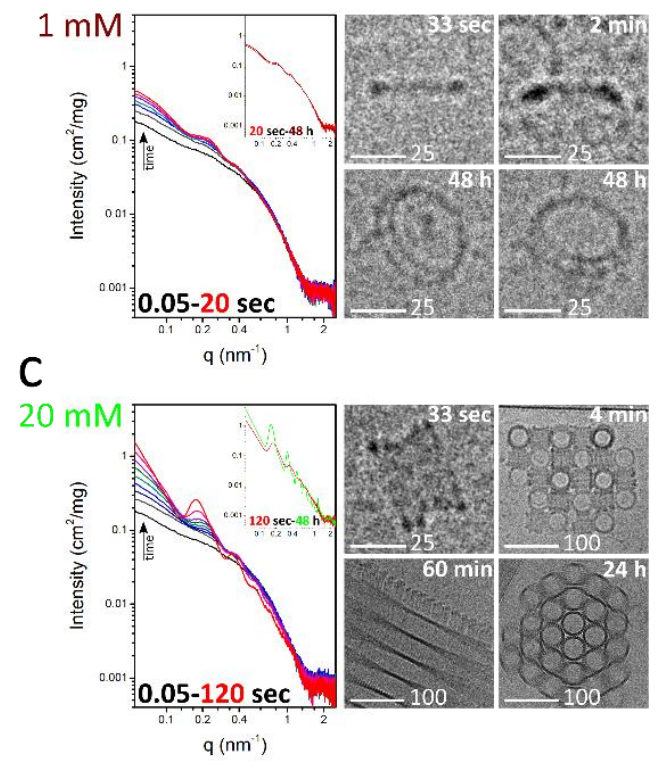

$\mathrm{e}$

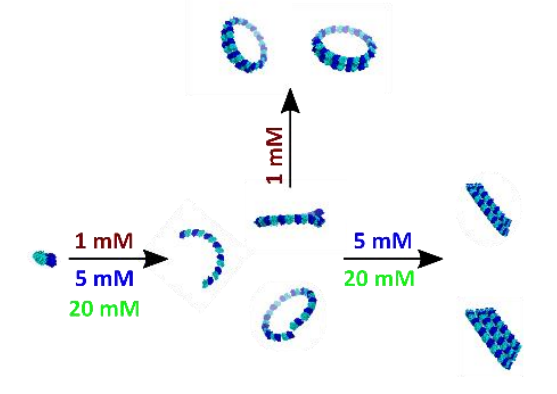

b

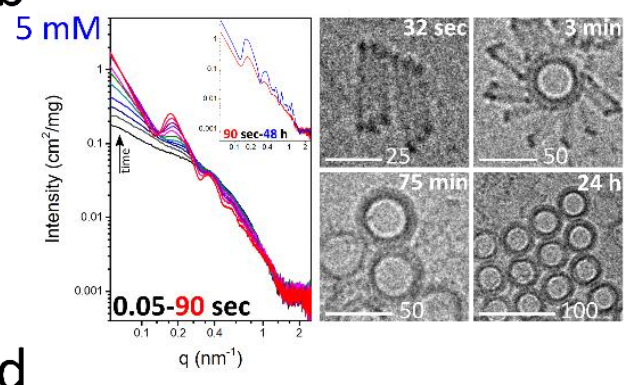

d

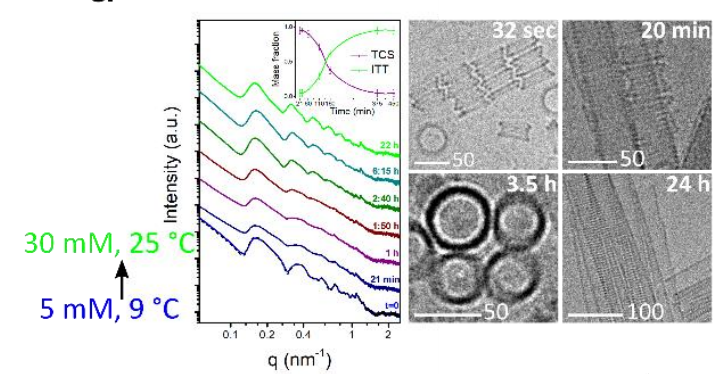

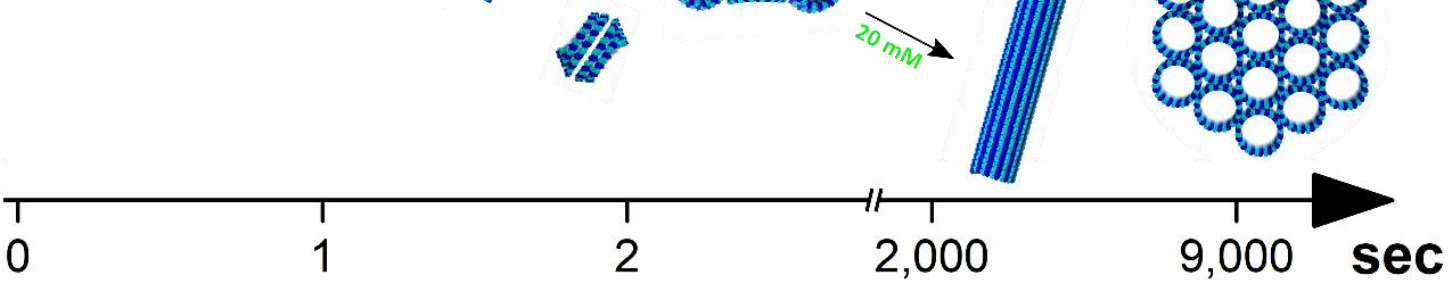

Figure 3| Assembly pathways of THPs, TCS/Ts and ITTs in the presence of spermine. a-c. Buffer-subtracted time-resolved synchrotron SAXS and time-resolved cryo-TEM results. A cold $\left(9^{\circ} \mathrm{C}\right)$ solution of $4 \mathrm{mg} / \mathrm{ml}$ tubulin reacted with $1 \mathrm{mM}(\mathbf{a}), 5$ $\mathrm{mM}(\mathbf{b})$, or $20 \mathrm{mM}$ (c) spermine. The first SAXS curve in each panel corresponds to 4 $\mathrm{mg} / \mathrm{ml}$ tubulin with no added spermine (black curve). The initial SAXS measurement at each spermine concentration was taken $50 \mathrm{msec}$ after mixing the tubulin and spermine solutions. The insets compare synchrotron SAXS data at $\mathrm{t} \cong 48 \mathrm{~h}$ with the last kinetic measurement that showed structural changes (at $\mathrm{t} \cong 20,90$, and $120 \mathrm{sec}$ with 1 , 5 and $20 \mathrm{mM}$ spermine, respectively). Cryo-TEM images were taken at different time 
points along the course of the reactions, as indicated. Scale bars are in nm units. $\mathbf{d}$. Transformation of TCST bundles into ITT bundles. A solution of $4 \mathrm{mg} / \mathrm{ml}$ tubulin was incubated for $48 \mathrm{~h}$ at $9{ }^{\circ} \mathrm{C}$ with $5 \mathrm{mM}$ spermine and mainly TCSTs formed. At that time $(t=0)$, the spermine concentration was increased to $30 \mathrm{mM}$ and the temperature was increased to $25{ }^{\circ} \mathrm{C}$. Synchrotron SAXS and cryo-TEM measurements were then performed as a function of time. The inset shows the mass fractions of TCS structures and ITT as a function of time, based on the analysis, shown in Extended data Fig. 3c. As a control, tubulin was incubated with $5 \mathrm{mM}$ spermine for $48 \mathrm{~h}$ at $9{ }^{\circ} \mathrm{C}$ and then only the temperature was raised to $25{ }^{\circ} \mathrm{C}$ for additional $48 \mathrm{~h}$ (black broken curve). $\mathbf{e}$. Schematic illustration of tubulin assemblies as a function of incubation time and spermine concentration. Cartoons are not to scale.

\section{Structural analysis}

The scattering curves from models of THPs, TCS/Ts and ITTs were computed and fitted to the data by D+ software (https://scholars.huji.ac.il/uriraviv/book/d-0) ${ }^{32}$, developed in our lab. The models were obtained by docking the atomic structure of the tubulin dimer (PDB ID 3J6F ${ }^{33}$ ) with a solvation layer (see Methods), into the assembly symmetry of each structure. The assembly symmetry describes the translation and rotation of each tubulin dimer in the structure. The models are illustrated in Fig. 4a-c. The SAXS fit to the THP phase was obtained by comparing the computed THP models with the $4 \mathrm{mg} / \mathrm{ml}$ tubulin and $1 \mathrm{mM}$ spermine kinetics and steady-state measurements (Fig. 4a). 1, 2, and 18 dimers were placed in a symmetry of a truncated conical spiral (see Methods Eq. 1), as representative models for small and relatively large tubulin assemblies. The THPs model is defined by the initial (largest) and final (smallest) radii, the helical pitch, and the number of helical turns between the two radii (see Methods 
Eq. 1). SAXS and cryo-TEM analyses revealed that radii polydispersity should be considered in the models (Figure 4). Hence, we assumed a Gaussian distribution with a standard deviation of $1.3 \mathrm{~nm}$ around the average initial radius. Each signal in the 1 $\mathrm{mM}$ spermine reaction in Fig. 4a, was fitted to a linear combination of the models (Extended Data Fig 8a). The best fitted model of THP, comprised 18 dimers, had an initial radius of $21.1 \pm 1.3 \mathrm{~nm}$, a final radius of $17.2 \pm 1.1 \mathrm{~nm}$, a helical pitch of $5.7 \pm 0.5$ $\mathrm{nm}$, and completed 1.5 helical turns between the initial and final radii.

a

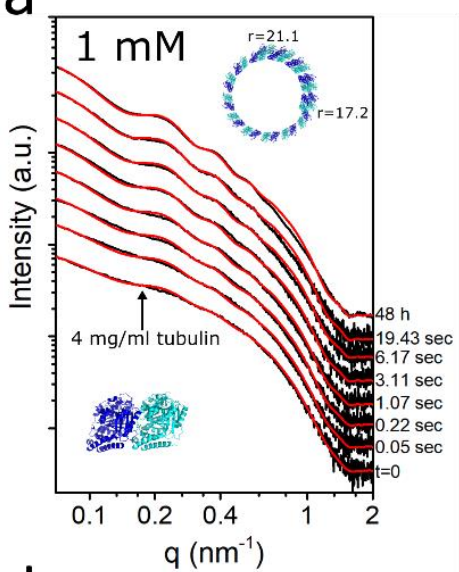

d

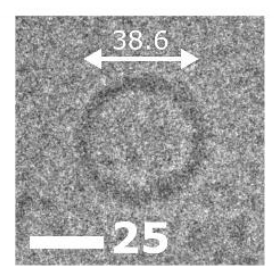

g

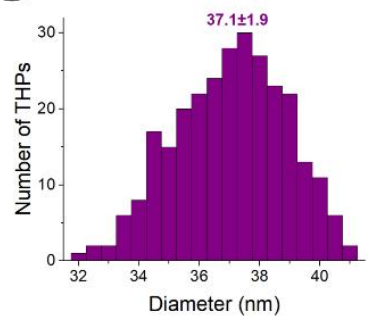

b

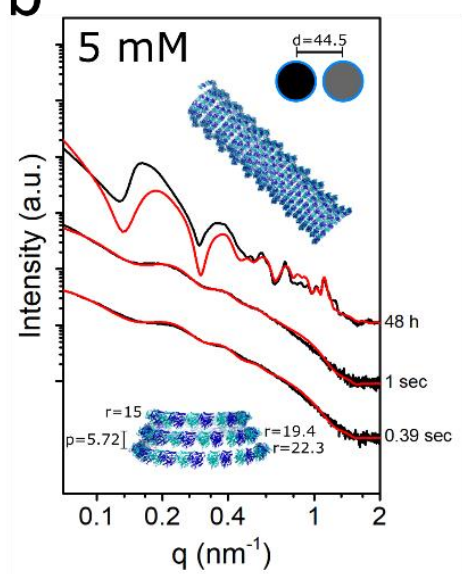

e

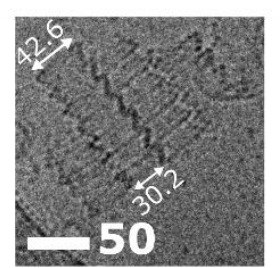

$\mathrm{h}$

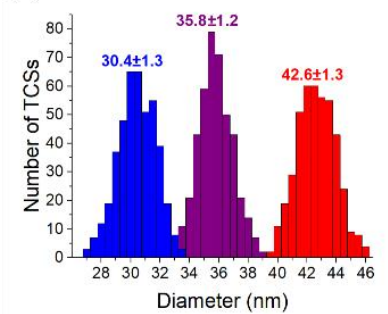

C

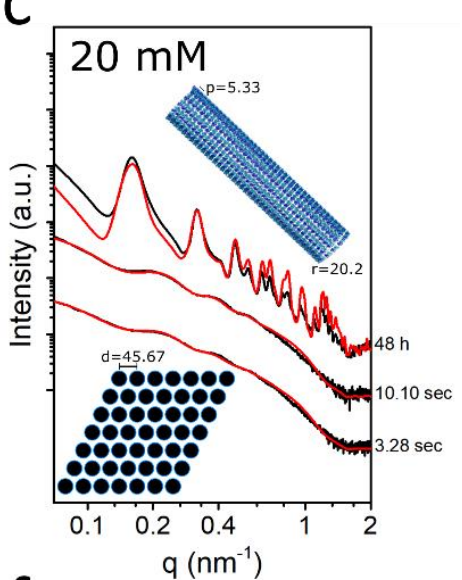

f

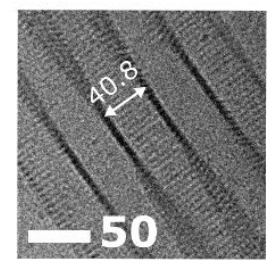

i

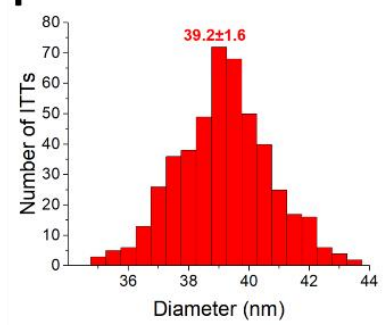

Figure 4| Models of THPs, TCS/Ts and ITTs. a-c. Supernatant-subtracted synchrotron SAXS and buffer-subtracted time-resolved synchrotron SAXS data (black curves), and computed SAXS curves (red curves) of THPs, TCSs, TCSTs, and ITTs models. All the models were convolved with a Gaussian instrument resolution function with standard deviation of $0.01 \mathrm{~nm}^{-1} .{ }^{34}$ In the THP model (a, b $0.39 \mathrm{sec}, \mathbf{c} 3.28 \mathrm{sec}$ ) the 
radius, $r$, continuously decreased from $21.1 \pm 1.3$ to $17.2 \pm 1.1 \mathrm{~nm}$, the pitch, $p$, was 5.72 $\mathrm{nm}$, and 18 dimers formed 1.5 helical turns. To fit the data, we also added THP fragments containing 1 and 2 dimers (the mass fractions of the models are shown in Extended Data Fig. 8a, b, e). In the TCS model (b $1 \mathrm{sec}, \mathbf{c} 10.10 \mathrm{sec}), r$ continuously decreased from $22.3 \pm 1.4$ to $15.0 \pm 0.9 \mathrm{~nm}, p$ was $5.72 \mathrm{~nm}$, and 32 dimers formed 2.5 helical turns. To fit the data, the TCS model was added to the THP models (the mass fractions of the models are shown in Extended Data Fig. 8c, f). In the TCSTs model (b, $48 \mathrm{~h}$ ), $r$ continuously decreased from 22.3 to $15.0 \mathrm{~nm}$ and then increased back to 22.3 $\mathrm{nm}$ (see text), $p$ was $5.72 \mathrm{~nm}$, and the center to center distance between two anti-parallel tubes was $44.5 \mathrm{~nm}$. In the model of ITT hexagonal bundle (c, $48 \mathrm{~h}), r$ was $20.2 \mathrm{~nm}, p$ was $5.33 \mathrm{~nm}$, the center to center distance between helical tubes was $45.67 \mathrm{~nm}$, and the bundle contained 49 tubes. Schematic illustrations of the models are also shown. Black circles represent the lattice of the TCSTs and ITTs models, the change in color of the circles (in b) represents the anti-parallel alignment. d-f. Cryo-TEM images of THPs (d), TCSTs (e), and ITTs (f). g-i. On the basis of our cryo-TEM images we derived the diameter distributions of THPs (g, 279 assemblies were measured), TCS subunits (h, 431) and ITT (i, 476). The numbers in the figure are nm units.

The fit to the TCS phase (Fig. 4b) was obtained by comparing the above-mentioned computed models (THP models) for the early time-resolved measurements $(t=0.39 \mathrm{sec}$ in Fig. 4b, mass fraction shown in Extended Data Fig. 8b), THP models with TCS subunit model for a later measurement ( $t=1 \mathrm{sec}$ in Fig. 4b, mass fraction shown in Extended Data Fig. 8c), and two antiparallel TCSTs for the steady state measurement ( $\mathrm{t}=48 \mathrm{~h}$ in Fig. $4 \mathrm{~b}$ ). The TCS subunit was built from 32 dimers by extending the helical geometry and dimer orientation of THP model (see Methods). Owing to the immediate 
interactions between TCS subunits, it was difficult to measure isolated subunits. We therefore selected the first SAXS signal from the assembly reaction with $5 \mathrm{mM}$ spermine that displayed significant scattering changes from the THP signal, in which the TCS was likely to be an intermediate product. To fit this signal, we added the 32 dimer TCS model to the linear combination of 1, 2, and 18 dimer THP models (Extended Data Fig. 8b-d). The TCS model (32 dimers) had an initial radius of 22.3 \pm 1.4 $\mathrm{nm}$, a final radius of $15.0 \pm 0.9 \mathrm{~nm}$, a helical pitch of $5.72 \pm 0.05 \mathrm{~nm}$, and 2.5 helical turns between the initial and final radii. The steady state signal (48 h) from $4 \mathrm{mg} / \mathrm{ml}$ tubulin with $5 \mathrm{mM}$ spermine was fitted to a model of two antiparallel TCSTs, built from monodispersed TCS subunits (with a continuously decreasing radius from $22.3 \pm 0.7$ to $15.0 \pm 0.7 \mathrm{~nm})$. The subunits were placed on top of each other and were connected by curved PFs with a continuously increasing radius from $15.0 \pm 0.7$ to $22.3 \pm 0.7 \mathrm{~nm}$, and a total vertical rise of $2.86 \pm 0.03 \mathrm{~nm}$ (half a helical pitch). This structure formed a continuous helical tube with a pitch of $5.72 \pm 0.05 \mathrm{~nm}$. The helical pitch value was evaluated according to the Bragg peak located at $q=\frac{2 \pi}{\text { pitch }}=1.098 \pm 0.015 \mathrm{~nm}^{-1}$. The fit of the models to the data was not as good when the tubes were built from stacks of TCS subunits (without the connecting curved PFs), or when the TCS subunit was built from a stack of three tubulin-rings (Extended Data Fig. 9). Nevertheless, we cannot rule out the possibility that TCSTs were assembled from a stack of TCSs. The antiparallel alignment of TCSTs was obtained by duplicating one tube and flipping its direction by $180^{\circ}$ vertically and horizontally. The center-to-center distances between tubes that best fitted the data were between 44.5 and $47 \mathrm{~nm}$. There are missing features in this model compared with the entire TCS phase, like the contribution of the various TCS associations (Fig. 1 e-g) as well as the 2D-cubic and quasi 2D-hexagonal lattices (Fig. 11-n). The hierarchical TCS populations and their different bundle morphologies pose 
a challenge to achieve a good fit. Yet, it is notable that the main population is TCSTs in an antiparallel alignment. The fit to the ITT phase (Fig. 4c) was obtained by comparing the THP model with the early time-resolved measurements $(\mathrm{t}=3.28 \mathrm{sec}$ in Fig. 4c, fraction ratio shown in Extended Data Fig. 8e), THP models with TCS subunit model with a later measurement $(t=10.10 \mathrm{sec}$ in Fig. 4c, mass fraction shown in Extended Data Fig. 8f), and a model of ITTs in a 2D-hexagonal lattice with the steady state scattering curve from $4 \mathrm{mg} / \mathrm{ml}$ tubulin with $20 \mathrm{mM}$ spermine ( $\mathrm{t}=48 \mathrm{~h}$ in Fig. $4 \mathrm{c}$ ). ITTs were built according to a helical symmetry (see Method Eq. 2). The best fitted model included a hexagonal bundle of $7 \times 7$ ITTs, each with a radius of $20.2 \pm 0.1 \mathrm{~nm}$, a helical pitch of $5.33 \pm 0.04 \mathrm{~nm}$, and a center-to-center distance of $45.67 \pm 0.07 \mathrm{~nm}$, corresponding to a tightly packed bundle.

Histograms of THP, TCS and ITT diameters were obtained on the basis of our cryoTEM images (Fig.4d-i). The mean diameters of the different structures obtained from the cryo-TEM analysis were consistent with our SAXS models.

Spermine biosynthesis starts with decarboxylation of ornithine to the polyamine putrescine $(2+)$ followed by enzymatic reactions to spermidine $(3+)$ and then to spermine $(4+)^{35}$. When tubulin was incubated with the polyamines putrescine or spermidine neither TCS-based assemblies nor ITT formed (Extended Data Fig. 10a-b). Tubulin was also incubated with thermospermine (a structural isomer of spermine in which one amino group, out of four, exchanged position with a neighboring methylene group, Extended Data Fig. 10c). When tubulin was incubated with 2.5-5 mM thermospermine, both SAXS and cryo-TEM show that TCS subunits were formed but did not assemble into the higher order TCS-based structures, and condensed nonspecific aggregates were present in the solutions (Extended Data Fig. 10d, e). At higher 
concentration, ITT started to form but thermospermine was less efficient in promoting ordered assemblies formation compared with spermine. The results show that minor changes in the chemical structure of spermine can have significant structural consequences.

So far GDP-tubulin was used. We have also preformed experiments in the presence of GTP or its nonhydrolyzable analog GMPPCP (Extended data Fig. 11a, b). The binding of these nucleotides to tubulin promotes MT assembly. THPs did not form at $1.5 \mathrm{mM}$ spermine or less, consistent with the fact that GTP induce disassembly of tubulin rings ${ }^{28}$. At higher spermine concentration, TCST and/or ITT assembled in the presence of all three nucleotides, and their mass fraction was weakly depended on nucleotide type (Extended data Fig. 11c, d).

Spermine and the MAP tau bind to overlapping amino acid stretches in the C-terminal domain of tubulin and with similar micromolar binding affinities but different binding stoichiometries $^{36}$. Other MAPs may interfere with tubulin-spermine interactions. To check the effect of MAPs, tubulin with MAPs were incubated with spermine at $9{ }^{\circ} \mathrm{C}$ for $48 \mathrm{~h}$. When the spermine concentration was between 1 and $5 \mathrm{mM}$, mainly THP and individual TCS subunits formed (Extended Data Fig. 12a, c). At $10 \mathrm{mM}$ spermine, individual TCS subunits coexisted with ITTs (Extended Data Fig. 12a, b, d). At higher spermine concentrations (15 - $20 \mathrm{mM}$ ), only ITTs formed (Extended Data Fig. 12a, e). We found that MAPs inhibited the vertical association of TCS subunits and suppressed the assembly of TCSTs.

\section{$\underline{\text { Outlook }}$}


This work revealed remarkable hierarchical tubulin assembly architectures and demonstrated the versatility of tubulin self-association. We showed the effect of millimolar spermine concentrations (between $1 \mathrm{mM}$ and $30 \mathrm{mM}$ ) on tubulin assembly, which induced tubulin - THPs - TCSs - TCSTs - bundles of antiparallel TCSTs - ITTs phase transitions. Each structure was hierarchically constructed from precursor (transient) assemblies, observed at lower spermine concentrations. At each spermine concentration, the final assembly products were stable for days at low temperature. Tubulin C-terminal domain was demonstrated to interact with spermine at 2:1 spermine/C-terminal tail stoichiometry, on two separated binding regions ${ }^{36}$. The increasing spermine/tubulin molar ratio and the multiple associations of the assemblies in our study (Figs. 1-3) imply that spermine has various potential binding sites, with varying affinities. The hierarchical architectures were shown to depend on the structure of spermine, indicating that specific ion bridges dictate the assembly morphology. Although there is currently no theory that can explain the hierarchical behavior of TCSbased assemblies, the self-association versatility of tubulin provides a fascinating means to design and create a wide range of building blocks for biomaterial applications. 


\section{Methods}

Materials were purchased from Sigma-Aldrich, unless otherwise indicate.

Tubulin purification. Tubulin was purified from porcine brains by three polymerization/depolymerization cycles $^{28}$. The first cycle was at low salt buffer, maintaining microtubule associated proteins (MAPs) to achieve low tubulin critical concentration and hence a higher yield ${ }^{37}$. Subsequent cycles were performed in a highmolarity buffer to eliminate MAPs from the final product ${ }^{38}$. The purified protein was aliquoted into Eppendorf tubes, flash frozen in liquid nitrogen, and stored at $-80{ }^{\circ} \mathrm{C}$.

Purification of Tubulin with MAPs. Porcine brain MT protein, consisting of approximately $80 \%$ tubulin and $20 \%$ MAPs, was isolated by two polymerization/depolymerization cycles at low salt buffers (a slight modification of a published protocol) $)^{37}$. In our purification, polymerization was done in $100 \mathrm{mM}$ MES, $1 \mathrm{mM}$ EGTA, $0.5 \mathrm{mM} \mathrm{MgCl} 2$ (Fluka) and $10 \%$ glycerol (J.T. Baker) at $\mathrm{pH}$ 6.6. The depolymerization was done in $150 \mathrm{mM}$ monosodium glutamate, $30 \mathrm{mM}$ sodium phosphate monobasic (Macron fine chemicals), at pH 6.7. 
Sample preparation. Stored tubulin was thawed on ice, after which three polymerization/depolymerization cycles (at $36^{\circ} \mathrm{C}$ and $0{ }^{\circ} \mathrm{C}, 30 \mathrm{~min}$ each) were performed to obtain GDP-rich tubulin (about $80 \%$ of the exchangeable sites contained GDP, determined using published protocols ${ }^{28,39}$ ). The tubulin was then centrifuged for $30 \mathrm{~min}$ at $15,700 \mathrm{~g}$ and $4{ }^{\circ} \mathrm{C}$. The top $85 \%$ supernatant was taken for the experiments. The samples were diluted to the required concentration $(8 \mathrm{mg} / \mathrm{ml})$ using PEM 50 buffer (50 mM PIPES, $1 \mathrm{mM} \mathrm{MgCl} 2,1 \mathrm{mM}$ EGTA and 5\% glycerol, at pH 6.9).

Tubulin-spermine specimens $-8 \mathrm{mg} / \mathrm{ml}$ tubulin was mixed with an equal volume of spermine tetrahydrochloride solution in PEM50 at twice the final experimental spermine concentrations. Steady state samples were incubated at $9{ }^{\circ} \mathrm{C}$ for about $48 \mathrm{~h}$ before measurements.

Tubulin-putrescine/spermidine/thermospermine specimens- tubulin was incubated with putrescine dihydrochloride, spermidine trihydrochloride or thermospermine hydrochloride (ChemCruz) as described above for spermine specimens.

GTP/GMPPCP-tubulin Specimens - $8 \mathrm{mg} / \mathrm{ml}$ tubulin was incubated at $36{ }^{\circ} \mathrm{C}$ for $45 \mathrm{~min}$ with $4 \mathrm{mM}$ of either GTP or GMPPCP. Equal volumes of tubulin and spermine solution were then mixed and incubated at $9{ }^{\circ} \mathrm{C}$ for $48 \mathrm{~h}$, as explained.

X-ray scattering. Synchrotron SAXS experiments were performed at ID02 beamline at ESRF (Grenoble) ${ }^{40}$ or at P12 EMBL beamline in PETRA III at DESY (Hamburg) ${ }^{41}$. $100 \mu \mathrm{l}$ tubulin-spermine steady-state samples were divided to 30 and $70 \mu 1$ in two Eppendorf tubes. The $30 \mu 1$ sample was measured (before centrifuge was applied). The $70 \mu \mathrm{l}$ sample was then centrifuged $\left(15,700 \mathrm{~g}, 9{ }^{\circ} \mathrm{C}, 30 \mathrm{~min}\right)$ and $30 \mu \mathrm{l}$ from the top of the supernatant were measured and served as background. The sup was also used for the structural analysis of the THPs. The buffer (PEM50) with the final spermine 
concentration was measured before and after each sample and used as background. Steady-state and time-resolved SAXS data were measured at the ID02 beamline. The sample-to-detector distance was $2.5 \mathrm{~m}$, the X-ray wavelength was $0.0995 \mathrm{~nm}$, and the $q$-range was between $q_{\min }=0.029 \mathrm{~nm}^{-1}$ and $q_{\max }=3.057 \mathrm{~nm}^{-1}$, where $q$ is the magnitude of the scattering vector. For each steady-state measurement, we manually injected a $30 \mu \mathrm{L}$ of sample into $2 \mathrm{~mm}$ thick quartz capillary flow-cell and between 15 and 30 frames were measured while the sample was flowing through the capillary. The exposure time was $50 \mathrm{msec}$ per frame and the scattered intensity was recorded on an FReLoN 4 M Kodak CCD detector.

Time-resolved measurements were performed by mixing $200 \mu \mathrm{lof} 8 \mathrm{mg} / \mathrm{ml}$ tubulin with $200 \mu \mathrm{l}$ of 2,10 , or $40 \mathrm{mM}$ spermine at $9{ }^{\circ} \mathrm{C}$, using BioLogic SFM-400 stoppedflow instrument and a $1.3 \mathrm{~mm}$ quartz capillary. 20 frames were measured in each reaction, and the exposure time was $20 \mathrm{msec}$ per frame. Each reaction was repeated several times, with various initial delays and intervals, to collect a large data set, covering between 0.05 and $145.28 \mathrm{sec}$ for $1 \mathrm{mM}$ spermine (4 reactions), 0.05-155.27 sec for $5 \mathrm{mM}$ spermine (6 reactions), and $0.05-250.05 \mathrm{sec}$ for $20 \mathrm{mM}$ spermine (5 reactions). The different time-resolved data sets of each reaction showed that repeated exposure to the X-ray beam triggered disassembly of relatively small tubulin structures $^{28}$ (tubulin oligomers, THPs or TCSs subunits), which slowed down the assembly kinetics. As at least two identical time sets (delays and intervals) were used in all of the reaction conditions ( $4 \mathrm{mg} / \mathrm{ml}$ tubulin with 1,5 , or $20 \mathrm{mM}$ spermine), we were able to compare the reaction rates. The reaction buffers (PEM50) with the desired spermine concentrations were measured before and after each reaction and served as background (for data reduction an average of $\sim 50$ frames from the buffer measurements were used). 
The SAXS data in Fig. 3d were measured at the P12 beamline. The sample-to-detector distance was $3 \mathrm{~m}$, the X-ray wavelength was $0.124 \mathrm{~nm}$, and the $q$-range was between $q_{\min }=0.025 \mathrm{~nm}^{-1}$ and $q_{\max }=7.25 \mathrm{~nm}^{-1}$. For each measurement, $30 \mu \mathrm{L}$ of sample was injected into a $1.7 \mathrm{~mm}$ thick quartz capillary flow-cell by an automated sample changer (Arinax BioSAXS, 2018 model) ${ }^{42} .30$ frames were recorded while the sample was flowing through the capillary. The acquisition time per frame was $50 \mathrm{msec}$, and the scattered intensity was collected by a single-photon 2D PILATUS $6 \mathrm{M}$.

Data reduction was performed by manually selecting and averaging between 10 and 30 frames for each sample and $\sim 50$ frames for the buffer measurements. The averaged background signals were then subtracted from the averaged sample signals.

The SAXS measurements in Extended Data Figs. 1c and 10 were performed in our Inhouse X-ray scattering setup ${ }^{43}$. The samples were prepared by transferring $80 \mu 1$ of the samples into $1.5 \mathrm{~mm}$ quartz capillaries and centrifuged at $6,081 \mathrm{~g}$, and $9{ }^{\circ} \mathrm{C}$ for $1 \mathrm{~h}$. The pellets were measured as well as the supernatants, which served as background.

Cryo-Transmission Electron microscopy. The samples were imaged using transmission electron microscopy at cryogenic temperatures (cryo-TEM). A droplet of $3 \mu \mathrm{l}$ from the sample was deposited on a 300-mesh copper Lacey grid (Ted Pella Ltd.) and blotted using Vitrobot Mark IV (FEI Co.). Ultrathin films, with a thickness varying between 20 and $300 \mathrm{~nm}$, were formed. Specimens were vitrified by rapid plunging into liquid ethane precooled with liquid nitrogen at controlled temperature and $100 \%$ relative humidity, using Vitrobot Mark IV (FEI Co.). The vitrified samples were then transferred to a cryo-specimen holder (Gatan model 626; Gatan Inc.). The samples were imaged at $-177^{\circ} \mathrm{C}$, using a Tecnai $\mathrm{G}^{2}$ Spirit Twin T-12 TEM (FEI Co.), operated at an 
acceleration voltage of $120 \mathrm{kV}$ in a low-dose mode. Images were recorded on a $4 \mathrm{~K} \times$ 4K FEI Eagle CCD camera in defocus value that varied between 2 and $4 \mu \mathrm{m}$.

Computed models. Tubulin-dimer subunit was taken from the middle dimer of the nine-dimer lattice of PDB ID $3 \mathrm{~J}_{6} \mathrm{~F}^{33}$ (available at the protein data bank). In all of our SAXS models, the dimer was surrounded by a solvation layer whose thickness was 0.28 $\mathrm{nm}$ and its electron density contrast with respect to the solvent was $30 \frac{e}{\mathrm{~nm}^{3}}$, determined by $\mathrm{D}+(\text { https://scholars.huji.ac.il/uriraviv/book/d-0 })^{32}$, using a probe radius of 0.14 $\mathrm{nm}^{32}$. The contribution to the scattering intensity owing to the overlaps of the hydration layers in our SAXS models was negligible (within the SAXS resolution).

The dimer was translated by the vector $(-10.2 \AA, 9.74 \AA$, $-2.31 \AA)$, that aligned the center of mass of the dimer to the origin. The dimer was rotated according to TaitBryan $^{44}$ rotation angles, $\alpha, \beta$, and $\gamma$, around the $\mathrm{x}, \mathrm{y}$, and $\mathrm{z}$ axes, respectively, from its orientation in the $\mathrm{MT}^{33}$, using the rotation matrix:

$$
\begin{aligned}
& A(\alpha, \beta, \gamma) \\
& =\left(\begin{array}{ccc}
\cos \beta \cos \gamma & -\cos \beta \sin \gamma & \sin \beta \\
\cos \alpha \sin \gamma+\cos \gamma \sin \alpha \sin \beta & \cos \alpha \cos \gamma-\sin \alpha \sin \beta \sin \gamma & -\cos \beta \sin \alpha \\
\sin \alpha \sin \gamma-\cos \alpha \cos \gamma \sin \beta & \cos \gamma \sin \alpha+\cos \alpha \sin \beta \sin \gamma & \cos \alpha \cos \beta
\end{array}\right)
\end{aligned}
$$

with $\alpha=87^{\circ} \pm 10^{\circ}, \beta=0^{\circ} \pm 10^{\circ}$, and $\gamma=130^{\circ} \pm 50^{\circ}$ for the THP and TCS/T models, and $\alpha=88^{\circ} \pm 10^{\circ}, \beta=0^{\circ} \pm 10^{\circ}$, and $\gamma=180^{\circ} \pm 50^{\circ}$ for the ITT model.

Tubulin dimer assemblies (THPs, TCS/Ts and ITTs) were constructed using D+ software (https://scholars.huji.ac.il/uriraviv/book/d-0) ${ }^{32}$ which computed the expected SAXS curves of the models, and exported a PDB file for each model.

D+ uses a hierarchical method for computing the solution scattering intensities of large supramolecular assemblies ${ }^{45}$ 
THP and TCS/T models were built based on truncated conical spiral symmetry ${ }^{46}$, described by

Equation 1:

$$
\begin{aligned}
& x=t_{j} \cdot r_{i} \cdot \cos \left(\theta \cdot t_{j}\right) \\
& y=t_{j} \cdot r_{i} \cdot \sin \left(\theta \cdot t_{j}\right) \\
& z=h \cdot\left(1-t_{j}\right) \\
& \theta=a \cdot\left(t_{j}-1\right) \\
& \alpha=0 \quad, \quad \beta=0 \quad, \quad \gamma=\frac{180}{\pi} \cdot \theta \\
& h=\frac{P}{1-\frac{r_{f}}{r_{i}}} \\
& p=\frac{P}{n} \\
& a=\frac{2 \pi \cdot n}{1-\frac{r_{f}}{r_{i}}} \\
& s\left(t_{i}\right)=\frac{1}{2} \cdot t_{i} \cdot \sqrt{1+r_{i}^{2} \cdot\left(1+a^{2} \cdot t_{i}^{2}\right)}+\frac{1+r_{i}^{2}}{2 a \cdot r_{i}} \cdot \sinh ^{-1}\left(\frac{a \cdot r_{i} \cdot t_{i}}{\sqrt{1+r_{i}^{2}}}\right)
\end{aligned}
$$

The first tubulin dimer was placed using $t_{j}=t_{i}$. The following dimers were placed by decreasing $t_{j}$ using binary search to find $t$ value that solved the equation:

$$
\begin{aligned}
& s(t)=s\left(t_{i}\right)-l \cdot j \\
& t_{i}=1, t_{\text {min }}=\frac{r_{f}}{r_{i}}
\end{aligned}
$$


where $t_{\min } \leq t_{j} \leq 1$ and represents the $j$-th tubulin dimer after the initial dimer, $r_{i}$ and $r_{f}$ are the initial and final radii of the conical spiral (the largest and smallest radii, respectively), $n$ is the number of helical turns between the initial and final radii, $h$ is the height of a conical spiral form $t=0$ to $t=1, a$ is the number of helical turns (in radians) in a conical spiral of height $h, P$ is the vertical distance between the initial and final radii, $p$ is the helical pitch, $l$ is the distance between tubulin dimers along the conical spiral, and $s(t)$ is the arc length as a function of $t$.

The distance between the center of neighboring dimers along the helical arc $(l)$ was $9.18 \mathrm{~nm}$ (corresponds to the distance between dimers in a tubulin-ring containing 13 dimers with a radius of $19 \mathrm{~nm}$ from the origin to the center-of-mass of the dimers).

TCSTs model was built using TCS subunits which were placed one on top of the other with a vertical repeat distance. The TCS subunits were connected to form a continuous helical tube by a curved PF which is based on the same symmetry as above, except:

$$
\begin{gathered}
z=h \cdot\left(t_{j}-t_{\text {min }}\right) \\
\theta=a \cdot\left(t_{j}-t_{\text {min }}\right) \\
s(t)=s\left(t_{\text {min }}\right)+l \cdot j
\end{gathered}
$$

The radii polydispersity of the THPs and TCSs SAXS models was calculated according to a Gaussian distribution around an average initial radius. Each dimer in the THPs or TCSs was placed in a different radius, determined by the initial and final radii $\left(r_{i}, r_{f}\right)$, the vertical distance $(P)$, and number of helical turns between the two radii $(n)$. To sample the radii polydispersity, $P, n$ and $l$ were kept constant whereas the initial radius, $r_{i}$, was varied 15 times weighted by a normalized Gaussian distribution. The final radius for each iteration, $r_{f}^{\prime}$, was determined by using the equation: 


$$
r_{f}^{\prime}=\frac{r_{i}^{\prime}}{r_{i}} \cdot r_{f}
$$

where $r_{i}^{\prime}$ is the sampled initial radius. For each $r_{i}^{\prime}$ we calculated the expected SAXS curve, scaled it according to the normalized Gaussian distribution and averaged the curves.

The ITT model was built based on a helical symmetry ${ }^{24,28}$, described by Equation 2:

$$
\begin{aligned}
x & =r \cdot \cos \left(\theta_{i}\right) \\
y & =r \cdot \sin \left(\theta_{i}\right) \\
z & =\frac{p}{n_{\text {dimers }}} \cdot i \\
\theta_{i} & =\frac{2 \pi}{n_{\text {dimers }}} \cdot i \\
\alpha=0 \quad, \quad \beta & =0 \quad \gamma=\frac{180}{\pi} \cdot \theta_{i}
\end{aligned}
$$

where $r$ is the radius, $i$ represents the $i$-th tubulin dimer, $p$ is the helical pitch, $n_{\text {dimers }}$ is the number of dimers in one pitch.

For experimental SAXS data that were fitted by several models (THP and TCS), the fit was obtained by minimizing the following loss function with respect to $\chi_{i}, a$, and $C_{0}$, using a Matlab software:

$$
\text { Rasidual }=\sum_{q}\left|\left\langle I_{\text {signal }}(q)\right\rangle-\left(a \sum_{i} \chi_{i} I_{i}^{\text {model }}(q)\right)+C_{0}\right|
$$


where $\left\langle I_{\text {signal }}(q)\right\rangle$ is the average measured steady state signal (kinetics signals were not averaged), $a$ and $C_{0}$ are general scale and constant parameters, $I_{i}^{\text {model }}$ is the computed SAXS curve of the $i$-th model, and $\chi_{i}$ is the mass fraction of tubulin in that model.

Cartoons were created by $\mathrm{PyMOL}^{47}$ using the atomic coordinates exported from D+ software. The cartoons were constructed in D+ using the middle dimer of the ninedimer lattice of PDB ID $3 \mathrm{~J}_{6} \mathrm{~F}^{33}$ as the subunit.

\section{References}

1. Mitchison, T. \& Kirschner, M. Dynamic instability of microtubule growth. Nature 312, 237-242 (1984).

2. Kirschner, M. \& Mitchison, T. Beyond self-assembly: From microtubules to morphogenesis. Cell 45, 329-342 (1986).

3. Nicholson, W. V, Lee, M., Downing, K. H. \& Nogales, E. Cryo-electron microscopy of GDP-tubulin rings. Cell Biochem. Biophys. 31, 175-183 (1999).

4. Löwe, J., Li, H., Downing, K. H. \& Nogales, E. Refined structure of $\alpha \beta$-tubulin at $3.5 \AA$ A resolution. J. Mol. Biol. 313, 1045-1057 (2001).

5. Vater, W. \& Therapy, E. Structural diversity and dynamics of microtubules and polymorphic tubulin assemblies. Electron Microsc Rev. 3, 355-395 (1990).

6. Igaev, M. \& Grubmüller, H. Microtubule assembly governed by tubulin allosteric gain in flexibility and lattice induced fit. Elife 7, 1-21 (2018).

7. Wang, H.-W. \& Nogales, E. Nucleotide-dependent bending flexibility of tubulin regulates microtubule assembly. Nature 435, 911-915 (2005). 
8. Díaz, J. F., Pantos, E., Bordas, J. \& Andreu, J. M. Solution structure of GDPtubulin double rings to $3 \mathrm{~nm}$ resolution and comparison with microtubules. $J$. Mol. Biol. 238, 214-225 (1994).

9. Li, F. et al. Control of apoptosis and mitotic spindle checkpoint by survivin. Nature 396, 580-584 (1998).

10. Piperno, G. \& Fuller, M. T. Monoclonal antibodies specific for an acetylated form of alpha-tubulin recognize the antigen in cilia and flagella from a variety of organisms. J. Cell Biol. 101, 2085 LP - 2094 (1985).

11. Okabe, S. \& Hirokawa, N. Turnover of fluorescently labelled tubulin and actin in the axon. Nature 343, 479-482 (1990).

12. Tuszyński, J. A. et al. Molecular dynamics simulations of tubulin structure and calculations of electrostatic properties of microtubules. Math. Comput. Model. 41, 1055-1070 (2005).

13. Eagle, G. R., Zombola, R. R. \& Himes, R. H. Tubulin-Zinc Interactions : Binding and Polymerization. 221-228 (1983). doi:10.1021/bi00270a032

14. Sackett, D. L., Bhattacharyya, B. \& Wolff, J. Tubulin subunit carboxyl termini determine polymerization efficiency. J. Biol. Chem. 260, 43-45 (1985).

15. Mejillano, M. R. \& Himes, R. H. Assembly properties of tubulin after carboxyl group modification. J. Biol. Chem. 266, 657-664 (1991).

16. Serrano, L., de la Torre, J., Maccioni, R. B. \& Avila, J. Involvement of the carboxyl-terminal domain of tubulin in the regulation of its assembly. Proc. Natl. Acad. Sci. U. S. A. 81, 5989-5993 (1984).

17. Drechsel, D. N., Hyman, A. A., Cobb, M. H. \& Kirschner, M. W. Modulation of the dynamic instability of tubulin assembly by the microtubule-associated protein tau. Mol. Biol. Cell 3, 1141-1154 (1992). 
18. Mechulam, A. et al. Polyamine Sharing between Tubulin Dimers Favours Microtubule Nucleation and Elongation via Facilitated Diffusion. PLOS Comput. Biol. 5, e1000255 (2009).

19. Tabor, C. W. \& Tabor, H. 1,4-Diaminobutane (Putrescine), Spermidine, and Spermine. Annu. Rev. Biochem. 45, 285-306 (1976).

20. Suzuki, O., Matsumoto, T., Katsumata, Y. \& Oya, M. Rechtsmedizin A New Enzymatic Method for Quantitation of Spermine in Human Semen. Z Rechtsmed 88, 67-73 (1982).

21. Omine, M. \& Perry, S. Polyamine Content of AKR Leukemic Cells in Relation to the Cell Cycle. Cancer Res. 33, 2959-2964 (1973).

22. Pohjanpelto, P., Virtanen, I. \& Hölttä, E. Polyamine starvation causes disappearance of actin filaments and microtubules in polyamine-auxotrophic CHO cells. Nature 293, 475-477 (1981).

23. Anderson, P. J., Bardocz, S., Campos, R. \& Brown, D. L. The effect of polyamines on tubulin assembly. Biochem. Biophys. Res. Commun. 132, 147$154(1985)$.

24. Ojeda-Lopez, M. A. et al. Transformation of taxol-stabilized microtubules into inverted tubulin tubules triggered by a tubulin conformation switch. Nat. Mater. 13, 195 (2014).

25. Tang, J. X., Wong, S., Tran, P. T. \& Janmey, P. A. Counterion induced bundle formation of rodlike polyelectrolytes. Berichte der Bunsengesellschaft für Phys. Chemie 100, 796-806 (1996).

26. Needleman, D. J. et al. Higher-order assembly of microtubules by counterions: From hexagonal bundles to living necklaces. Proc. Natl. Acad. Sci. U. S. A. 101, 16099 LP - 16103 (2004). 
27. Webber, M. J., Appel, E. A., Meijer, E. W. \& Langer, R. Supramolecular biomaterials. Nat. Mater. 15, (2016).

28. Shemesh, A., Ginsburg, A., Levi-Kalisman, Y., Ringel, I. \& Raviv, U. Structure, Assembly, and Disassembly of Tubulin Single Rings. Biochemistry 57, 6153-6165 (2018).

29. Zhang, F. et al. Reentrant Condensation of Proteins in Solution Induced by Multivalent Counterions. Phys. Rev. Lett. 101, 148101 (2008).

30. Asor, R., Ben-Nun-Shaul, O., Oppenheim, A. \& Raviv, U. Crystallization, Reentrant Melting, and Resolubilization of Virus Nanoparticles. ACS Nano 11, 9814-9824 (2017).

31. de Vries, R. \& Cohen Stuart, M. Theory and simulations of macroion complexation. Curr. Opin. Colloid Interface Sci. 11, 295-301 (2006).

32. Ginsburg, A. et al. D+: software for high-resolution hierarchical modeling of solution X-ray scattering from complex structures. J. Appl. Crystallogr. 52, (2019).

33. Alushin, G. M. et al. High-Resolution Microtubule Structures Reveal the Structural Transitions in $\alpha \beta$-Tubulin upon GTP Hydrolysis. Cell 157, 1117 1129 (2014).

34. Ben-Nun, T., Ginsburg, A., Székely, P. \& Raviv, U. X+: a comprehensive computationally accelerated structure analysis tool for solution X-ray scattering from supramolecular self-assemblies. J. Appl. Crystallogr. 43, (2010).

35. Persson, L. Polyamine homoeostasis. Essays Biochem. 46, 11-24 (2009).

36. Lefèvre, J. et al. The $\mathrm{C}$ terminus of tubulin, a versatile partner for cationic molecules: binding of Tau, polyamines, and calcium. J. Biol. Chem. 286, 30653078 (2011). 
37. Farrell, K. W. \& Wilson, L. Tubulin-colchicine complexes differentially poison opposite microtubule ends. Biochemistry 23, 3741-3748 (1984).

38. Castoldi, M. \& Popov, A. V. Purification of brain tubulin through two cycles of polymerization-depolymerization in a high-molarity buffer. Protein Expr.

Purif. 32, 83-88 (2003).

39. Diaz, J. F. \& Andreu, J. M. Assembly of purified GDP-tubulin into microtubules induced by taxol and taxotere: Reversibility, ligand stoichiometry, and competition. Biochemistry 32, 2747-2755 (1993).

40. Van Vaerenbergh, P. et al. An upgrade beamline for combined wide, small and ultra small-angle x-ray scattering at the ESRF. AIP Conf. Proc. 1741, (2016).

41. Blanchet, C. E. et al. Versatile sample environments and automation for biological solution X-ray scattering experiments at the P12 beamline (PETRA III, DESY). J. Appl. Crystallogr. 48, 431-443 (2015).

42. Round, A. et al. BioSAXS Sample Changer: a robotic sample changer for rapid and reliable high-throughput X-ray solution scattering experiments. Acta Crystallogr. D. Biol. Crystallogr. 71, 67-75 (2015).

43. Nadler, M. et al. Following the structural changes during zinc-induced crystallization of charged membranes using time-resolved solution X-ray scattering. Soft Matter 7, 1512-1523 (2011).

44. Roberson, R. E. \& Schwertassek, R. Dynamics of multibody systems. (Springer Science \& Business Media, 2012).

45. Ginsburg, A. et al. Reciprocal Grids: A Hierarchical Algorithm for Computing Solution X-ray Scattering Curves from Supramolecular Complexes at High Resolution. J. Chem. Inf. Model. 56, 1518-1527 (2016).

46. Falcón, S. \& Plaza, Á. On the 3-dimensional k-Fibonacci spirals. Chaos, 
Solitons \& Fractals 38, 993-1003 (2008).

47. Schrödinger, L. Scholar (6). PyMOL Mol. Graph. Syst. Version 1., (2015).

\section{Acknowledgements}

We thank ESRF ID02 beamline (T. Narayanan and his team) and P12 beamline of the EMBL at DESY, Hamburg (D. Svergun and his team), as some of the data presented in the paper were acquired there. This project was supported by the Israel Ministry of Science, Israel Science Foundation (656/17), and by the United States-Israel Binational Science Foundation (2016311).

\section{Author contributions}

All authors designed the research, performed experiments, analyzed data, and wrote the paper.

\section{Author information}

The authors declare no competing financial interests. 

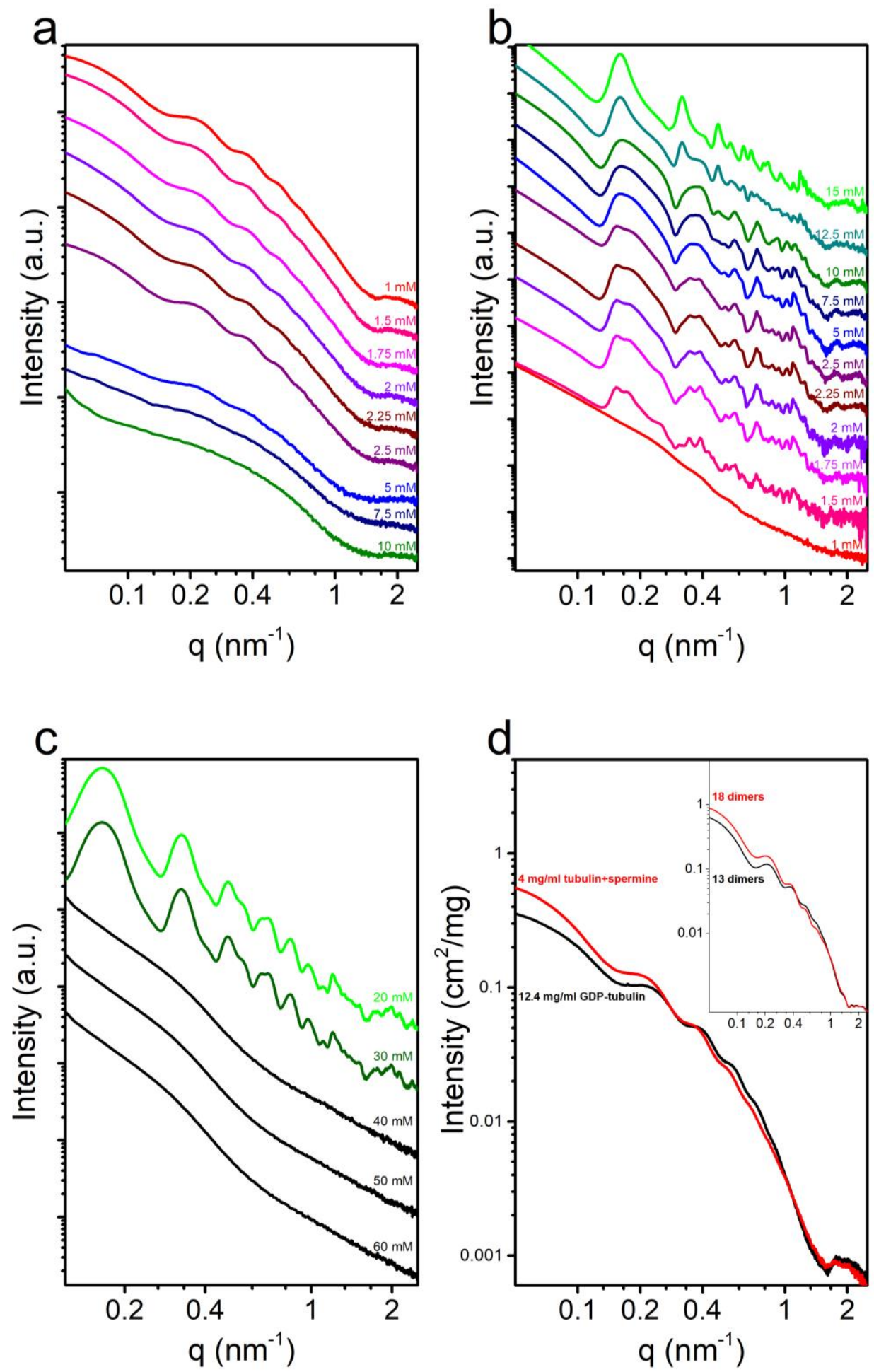

Figure 1| Spermine induced tubulin helical-pitch (THP), tubulin conical-spiral tubes (TCSTs) and inverted tubulin tubules (ITTs).

a. Azimuthally-averaged buffer-subtracted synchrotron SAXS curves from $4 \mathrm{mg} / \mathrm{ml}$ tubulin incubated at $9{ }^{\circ} \mathrm{C}$ for $48 \mathrm{~h}$ with 1 to $10 \mathrm{mM}$ spermine, as indicated. The solutions were centrifuged $\left(15,700 \mathrm{~g}, 9{ }^{\circ} \mathrm{C}, 30 \mathrm{~min}\right)$ and the measurements were performed on the supernatants and the buffer of the sample served as background. THPs were present in the samples that contained between 1 and $2.5 \mathrm{mM}$ spermine. At higher spermine 
concentrations, the concentration of THPs gradually decreased. b. Azimuthally-averaged supernatantsubtracted synchrotron SAXS curves from $4 \mathrm{mg} / \mathrm{ml}$ tubulin incubated at $9{ }^{\circ} \mathrm{C}$ for $48 \mathrm{~h}$ with 1 to $15 \mathrm{mM}$ spermine, as indicated. The solutions were centrifuged $\left(15,700 \mathrm{~g}, 9{ }^{\circ} \mathrm{C}, 30 \mathrm{~min}\right)$ and their supernatants served as background. At $1 \mathrm{mM}$ spermine, there were no significant differences between the sample and supernatant measurements (indicating that the structures in the sample were too small to sediment by the centrifugation). TCSTs bundles started to assemble at $1.5 \mathrm{mM}$ spermine. Above $5 \mathrm{mM}$ spermine, ITTs started to form and the concertation of TCSTs decreased. c. Azimuthally-averaged supernatant-subtracted SAXS curves from 4 $\mathrm{mg} / \mathrm{ml}$ tubulin incubated at $9{ }^{\circ} \mathrm{C}$ for $48 \mathrm{~h}$ with 20-60 mM spermine. At 20-30 mM spermine, ITT hexagonal bundles formed. Above $40 \mathrm{mM}$ spermine, however, ITT bundles or other assemblies did not form. We attribute the resolubilization of the assemblies to reentrant condensation ${ }^{29}$. d. Comparison between the scattering data at $9{ }^{\circ} \mathrm{C}$ from $4 \mathrm{mg} / \mathrm{ml}$ tubulin with $1 \mathrm{mM}$ spermine at steady state $(48 \mathrm{~h}$ ) and $12.4 \mathrm{mg} / \mathrm{ml} \mathrm{GDP}$-tubulin (without incubation), containing tubulin-rings ${ }^{27}$. A sharper decline in the intensity is observed with $1 \mathrm{mM}$ spermine. The inset compares the expected SAXS curve from a THP model, containing 18 dimers (red curve), with the expected scattering curve from tubulin ring containing 13 dimers. Similar differences are observed. 

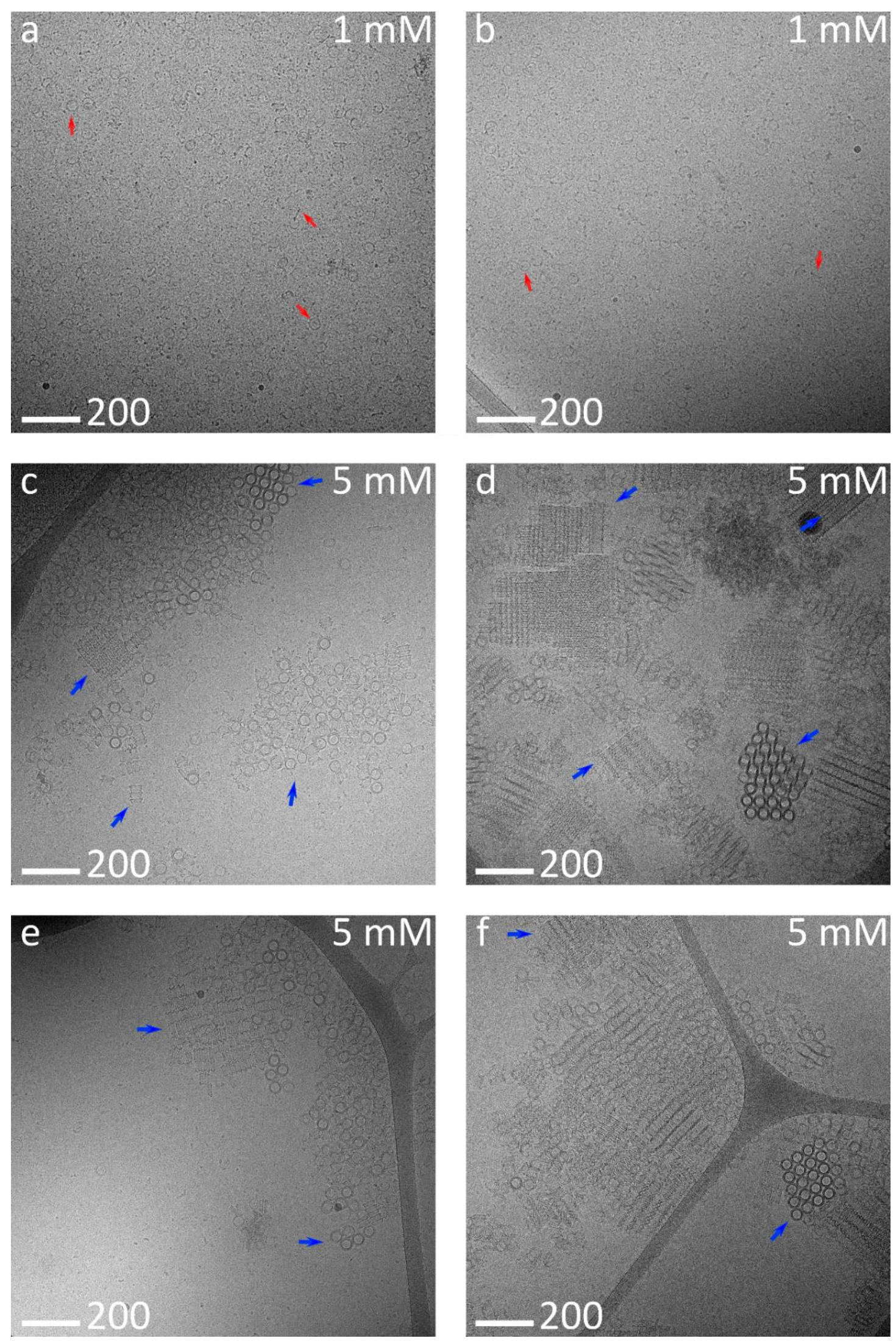

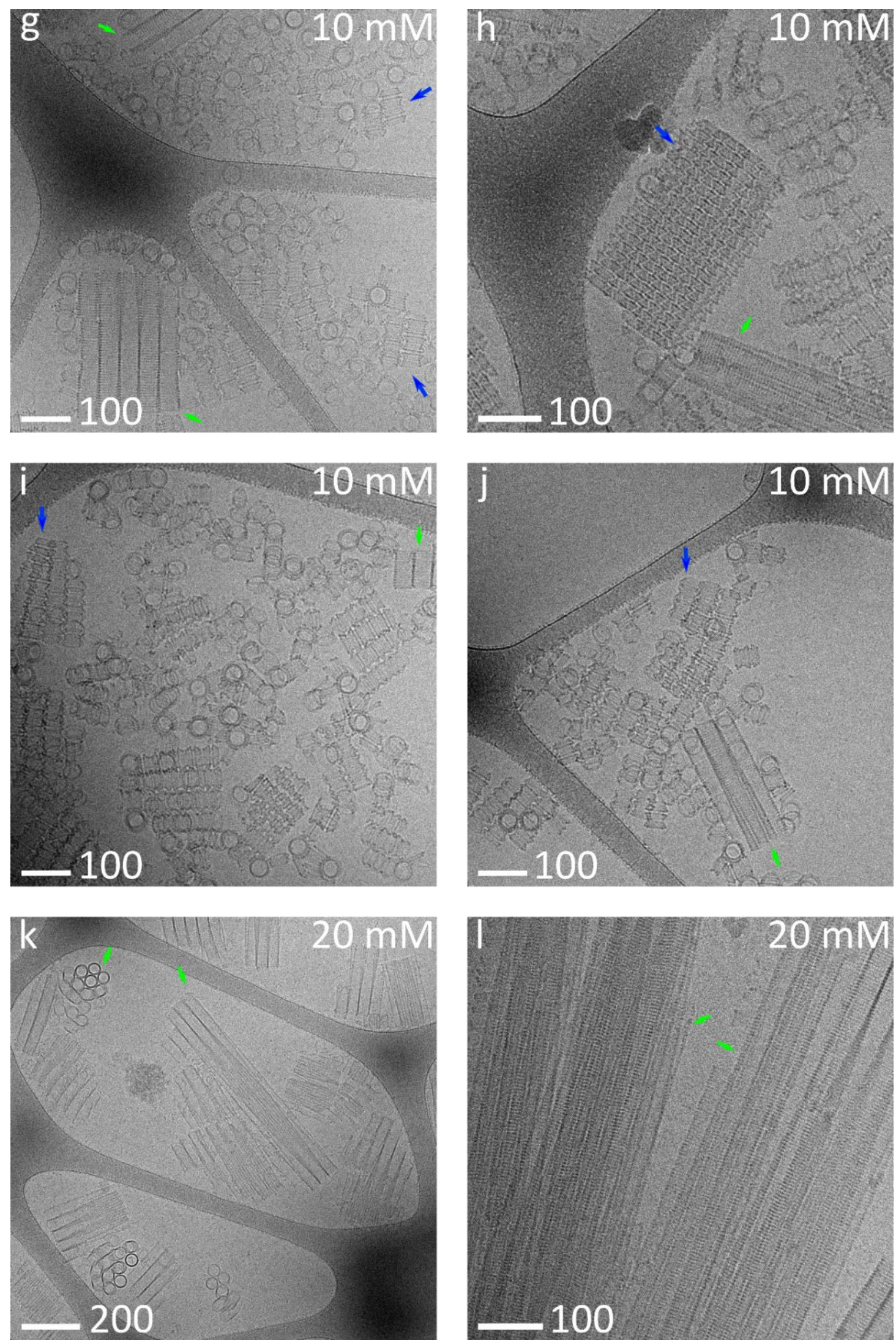

Figure 2| Cryo-TEM images of THP, TCST, and ITT.

Cryo-TEM images of $4 \mathrm{mg} / \mathrm{ml}$ tubulin incubated at $9{ }^{\circ} \mathrm{C}$ for $48 \mathrm{~h}$ with different spermine concentrations, as indicated. a, b. THP phase (red arrows) was observed in the $1 \mathrm{mM}$ spermine sample and contained mainly THPs and fragments of THPs (curved tubulin oligomers). c-f. TCS phase (blue arrows) was observed in the 5 mM spermine sample and contained the hierarchical TCS structures, in which TCSTs antiparallel bundles 
were the dominant population. g-j. TCSs and ITTs (green arrows) coexisted at $4 \mathrm{mg} / \mathrm{ml}$ tubulin with $10 \mathrm{mM}$ spermine. Base-to-base or top-to-top TCS associations were more abundant than base-to-top association (unlike $5 \mathrm{mM}$ spermine, in which top-to-base was more abundant). $\mathbf{k}$, l. ITT phase was observed in $4 \mathrm{mg} / \mathrm{ml}$ tubulin with $20 \mathrm{mM}$ spermine. This phase was more homogenous compared to TCS phase and contained mostly twisted ITTs hexagonal bundles. Scale bars are in nm units. 
a

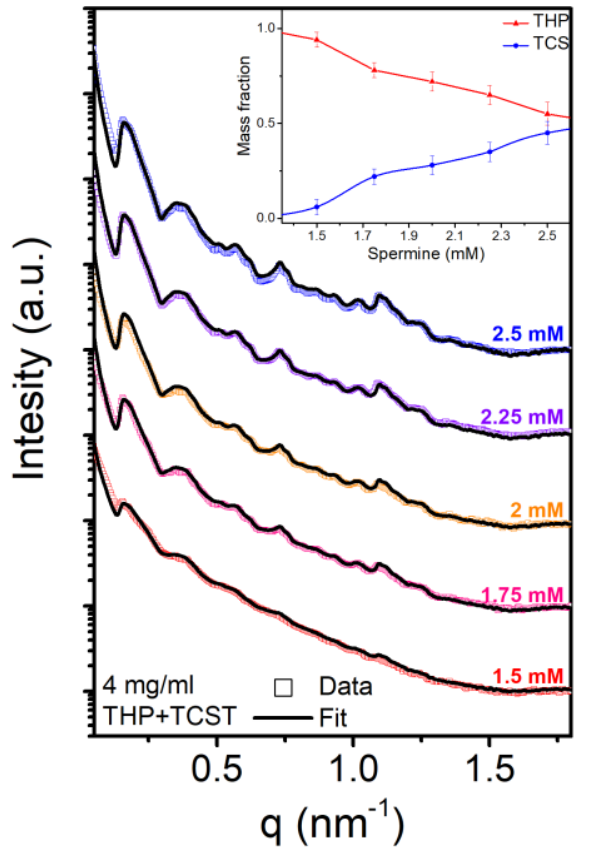

b

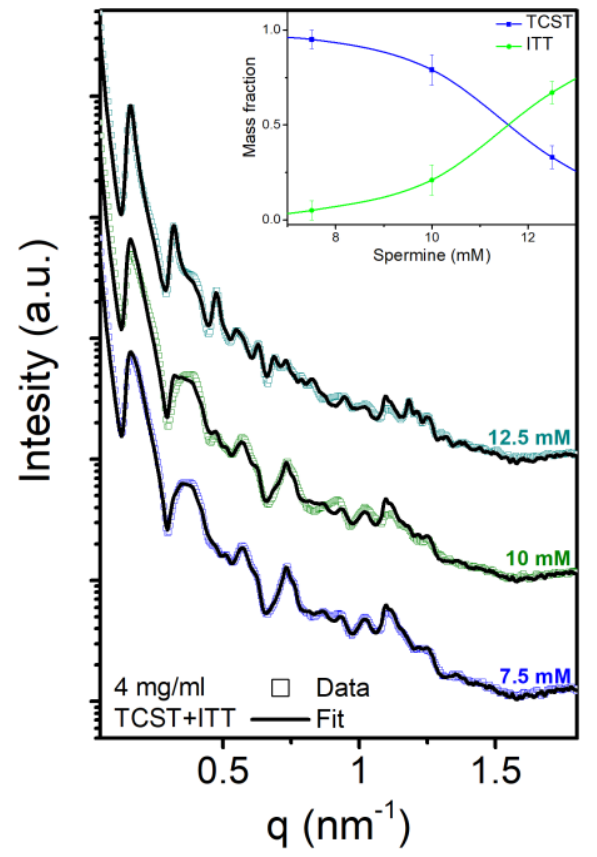

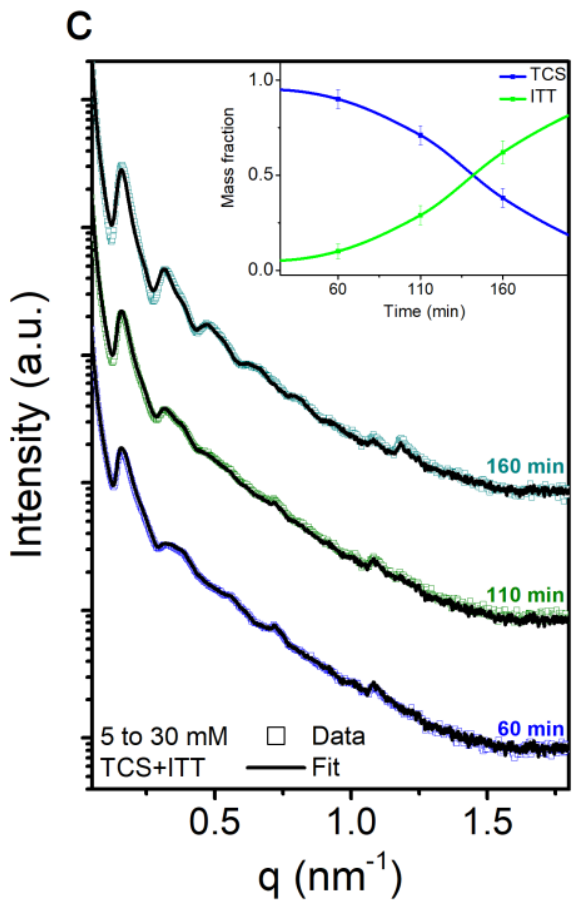

Figure 3| Coexistence of THP, TCS/T and ITT.

Azimuthally-averaged buffer-subtracted (a, c) or supernatant-subtracted (b) synchrotron SAXS curves (open square symbol), fitted to a linear combination (solid black curves) of experimental scattering curves that mostly contained THPs, TCS/Ts or ITTs. The insets show the mass fraction of the contributing assemblies. a. $4 \mathrm{mg} / \mathrm{ml}$ tubulin mixed with $1.5-2.5 \mathrm{mM}$ spermine. The fit was done by a linear combination of the $1 \mathrm{mM}$ spermine signal (THPs-rich) and the $5 \mathrm{mM}$ spermine signal (TCST-rich). b. $4 \mathrm{mg} / \mathrm{ml}$ tubulin mixed with 7.5, 10 or $12.5 \mathrm{mM}$ spermine. The fit was obtained by a linear combination of the $5 \mathrm{mM}$ spermine signal (TCSTrich) with the $15 \mathrm{mM}$ spermine signal (ITT-rich). c. Time resolved SAXS curves measured during the phase transition from TCST bundles to ITT bundles achieved by increasing spermine concentration from 5 to 30 $\mathrm{mM}$ (at $25^{\circ} \mathrm{C}$ ). The fit was done by a linear combination of the signal measured $21 \mathrm{~min}$ after addition of spermine (TCS-rich) with the signal measured $22 \mathrm{~h}$ after the addition of spermine (ITT-rich). 

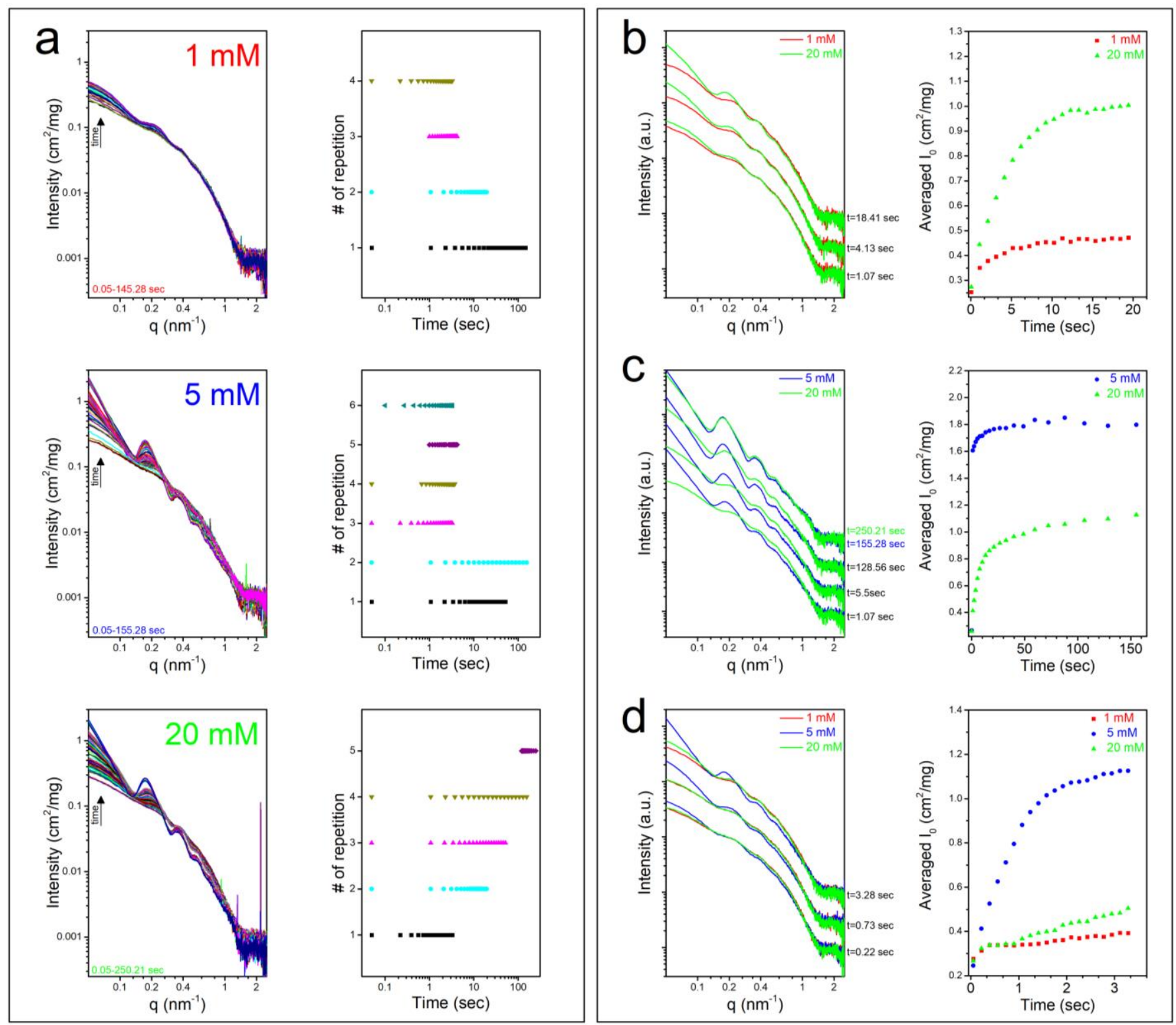

\section{Figure 4| Time resolved SAXS measurements.}

a. Azimuthally-averaged background-subtracted time-resolved synchrotron SAXS curves of $4 \mathrm{mg} / \mathrm{ml}$ tubulin with 1,5 , or $20 \mathrm{mM}$ spermine reactions. To collect sufficiently broad data set, each reaction was repeated several times, as shown on the plots next to the scattering curves. b-d. Comparsion between 1 and $20 \mathrm{mM}(\mathbf{b})$, 5 and $20 \mathrm{mM}(\mathbf{c})$, and 1,5 , and $20 \mathrm{mM}(\mathbf{d})$ reactions at the indicated times. The plots show that reactions with higher spermine concentrations pass through intermediates, formed in reactions with lower spermine concentations. On the right, the approximated intesities at $q \rightarrow 0, I_{0}$, are plotted as a fucntion time. $I_{0}$ corresponds to the average mass of the assemblies and was calculated by averaging the intensities between $q=0.05$ and $q=0.06 \mathrm{~nm}^{-1}$. The total growth of assemblies was slower in the 1 and $20 \mathrm{mM}$ spermine reactions, compared with the $5 \mathrm{mM}$ reaction. 


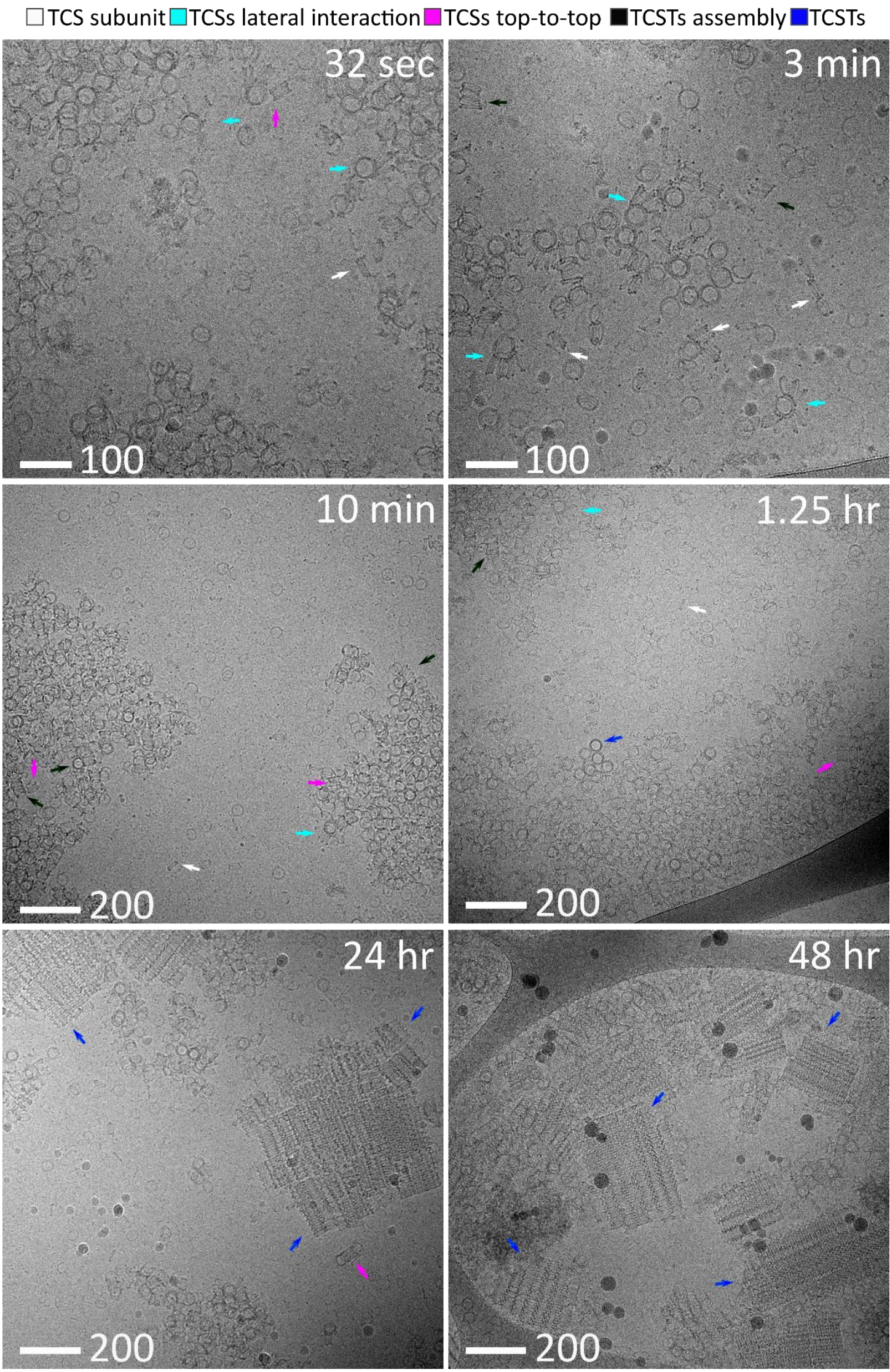

Figure 5| Time-resolved cryo-TEM images of TCSTs assembly.

$4 \mathrm{mg} / \mathrm{ml}$ tubulin was mixed with $5 \mathrm{mM}$ spermine at $9{ }^{\circ} \mathrm{C}$. Measurements were taken at the indicated times following the onset of the assembly reaction. $32 \mathrm{sec}$ to $3 \mathrm{~min}$ : TCS subunits (white arrows) formed and started to interact with each other in the vertical (top-to-top (magenta arrows) or base-to-top (TCST assembly, black 
arrows)) or lateral interactions (cyan arrows). $10 \mathrm{~min}$ : TCSs assembled into TCSTs by base-to-top associations. $1.25 \mathrm{~h}$ : TCSTs aggregated into antiparallel bundles (blue arrows). 24 to $48 \mathrm{~h}$ : highly ordered TCSTs in antiparallel bundles. All scale bars are in nm units. 
-THP $\square$ TCS subunit $\square$ TCSs top-to-top $\square$ TCSs lateral interaction $\square$ ITT

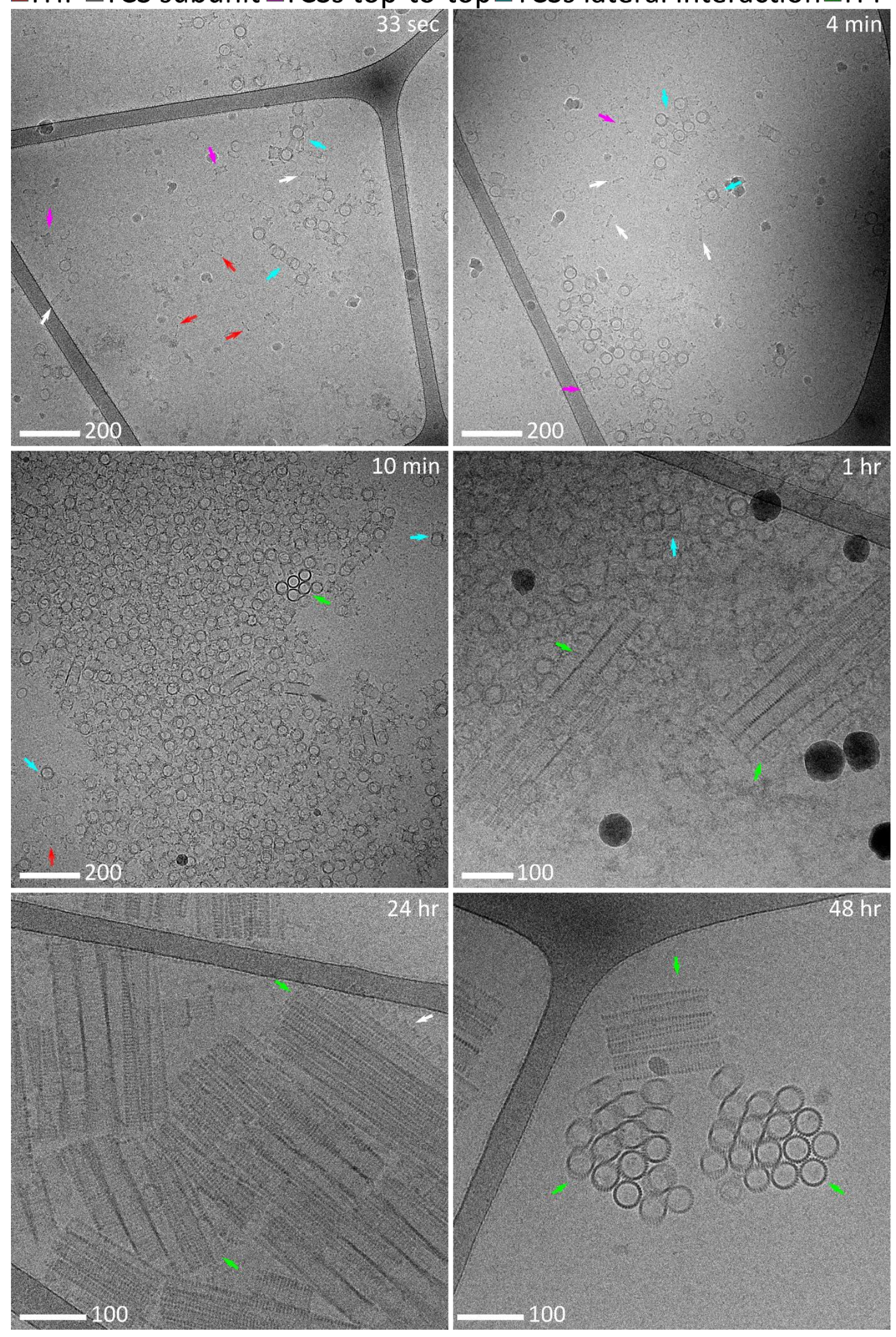

Figure 6| Time resolved cryo-TEM images of ITTs assembly.

$4 \mathrm{mg} / \mathrm{ml}$ tubulin was mixed with $20 \mathrm{mM}$ spermine at $9{ }^{\circ} \mathrm{C}$. Measurements were taken at the indicated times following the onset of the assembly reaction. 33 sec: THP (red arrows) and TCS subunits (white arrows) formed and started to interact with each other in vertical (top-to-top (magenta arrows) and lateral interactions 
(cyan arrows)). 4 min: TCSs mainly assembled by lateral associations. $10 \mathrm{~min}$ to $1 \mathrm{~h}$ : ITTs (green arrows) formed from TCSs subunits. 24 to $48 \mathrm{~h}$ : only highly ordered twisted ITT hexagonal bundles were observed. Scale bars are in nm units. 


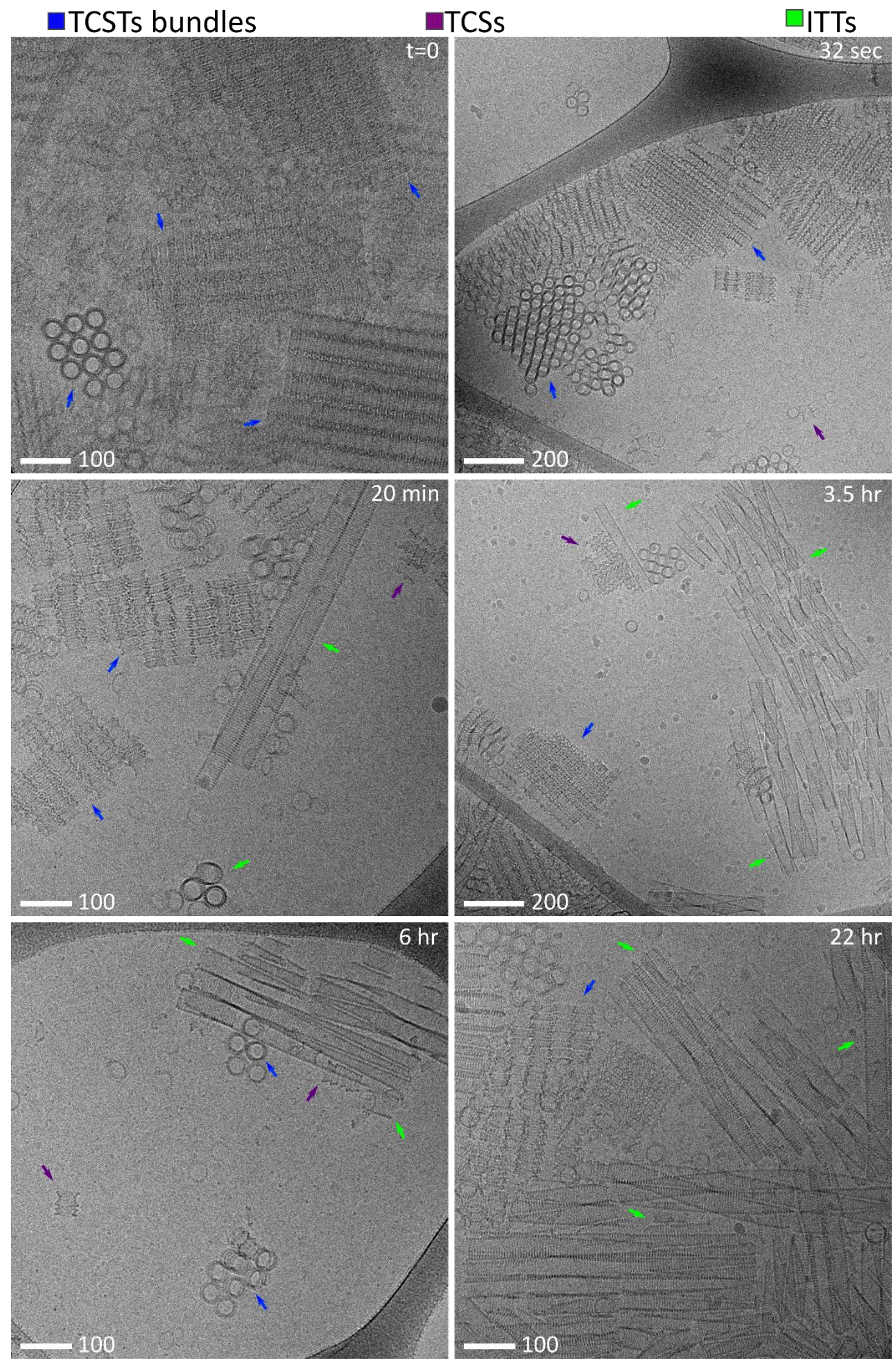

Figure 7| Time-resolved cryo-TEM of the transition between TCST bundles to twisted ITT hexagonal bundles.

$4 \mathrm{mg} / \mathrm{ml}$ tubulin was incubated with $5 \mathrm{mM}$ spermine at $9{ }^{\circ} \mathrm{C}$ for $48 \mathrm{~h}$. The solution was then exposed to $30 \mathrm{mM}$ spermine at $25^{\circ} \mathrm{C}$ and measurements were taken at different time points after the exposure to $30 \mathrm{mM}$ spermine, 
as indicated. $\mathrm{t}=0$ to $32 \mathrm{sec}$ : highly ordered TCSTs in massive antiparallel bundles (blue arrows). 20 min to 6 h: most of the TCSTs disassembled to TCS subunits (purple arrows), which gradually tranformed into ITT (green arrows). $22 \mathrm{~h}$ : the main population was bundles of ITTs, whereas some TCST bundles remained trapped. Scale bars are in nm units. 

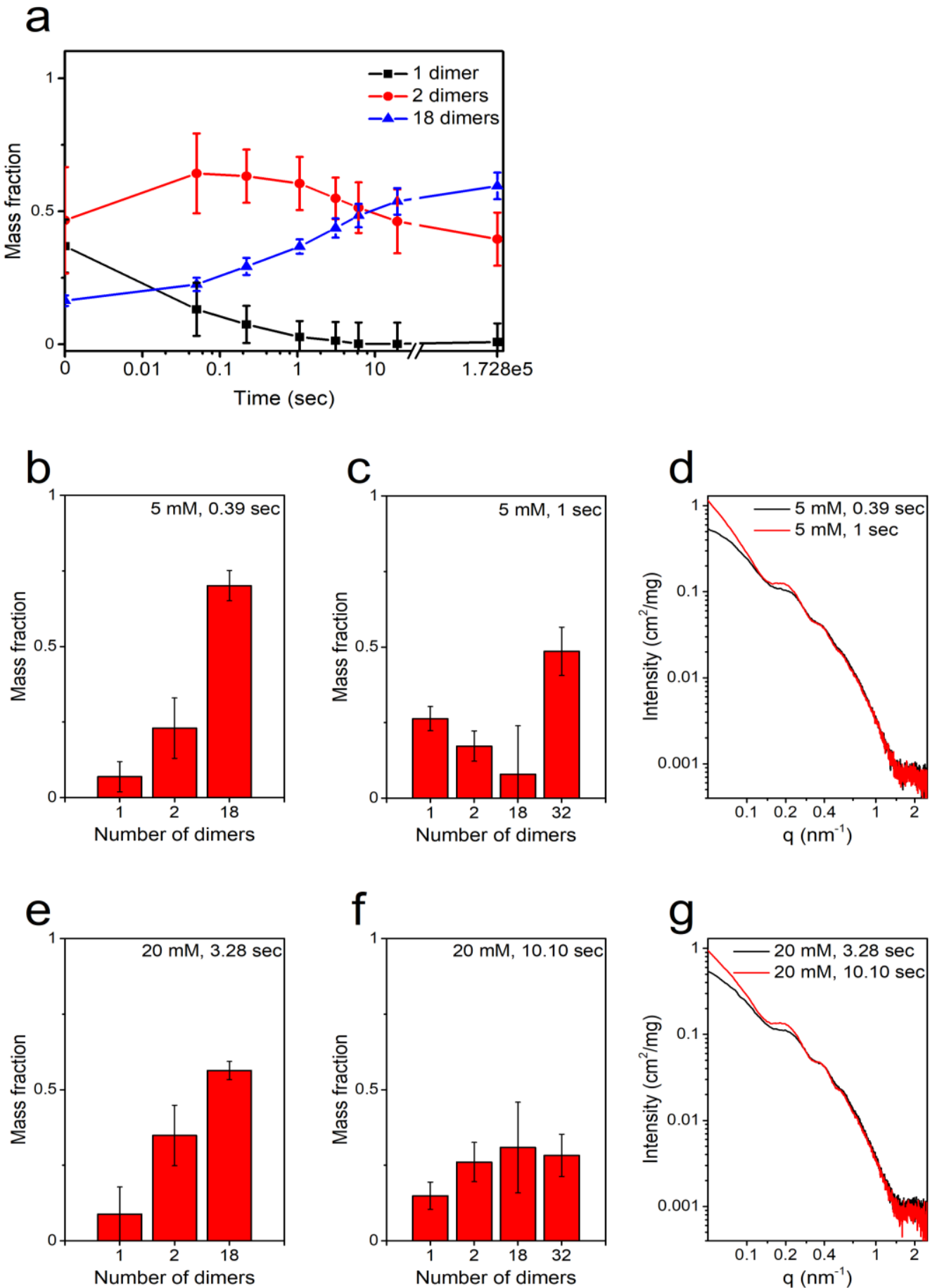

Figure 8| THP and TCS intermediate mass fraction distributions.

a. Kinetics and steady state measurements of $4 \mathrm{mg} / \mathrm{ml}$ tubulin with $1 \mathrm{mM}$ spermine (Fig. 4a in the main text) were fitted to the expected scattering curves from atomic models of THP containing 18 dimers and THP fragments containing 1 and 2 dimers. These models were selected as representative models for relatively large and small tubulin assemblies. We present the best fitted mass fraction distribution at the indicated time points after mixing tubulin with $1 \mathrm{mM}$ spermine. The results show that before adding spermine, the main population comprised small tubulin oligomers. As spermine was added the population shifted to larger tubulin assembly, 
as expected. b-d. The scattering curve from $4 \mathrm{mg} / \mathrm{ml}$ tubulin, $0.39 \mathrm{sec}$ after mixing with $5 \mathrm{mM}$ spermine (Fig. $4 \mathrm{~b}$ in the main text), was fitted to THP models (b). The scattering curve from $4 \mathrm{mg} / \mathrm{ml}$ tubulin, $1 \mathrm{sec}$ after mixing with $5 \mathrm{mM}$ spermine (Fig. $4 \mathrm{~b}$ in the main text), significantly differed from the curve at $0.39 \mathrm{sec}$ (dominated by THP structures) (d). The $1 \mathrm{sec}$ curve was fitted to TCS subunit model (32 dimer) in addition to the THP models (c). The best fitted mass fraction distribution contained mainly 32 dimers. e-g. The scattering curve from $4 \mathrm{mg} / \mathrm{ml}$ tubulin, $3.28 \mathrm{sec}$ after mixing with $20 \mathrm{mM}$ spermine (Fig. $4 \mathrm{c}$ in the main text), was fitted to THP models (e). The scattering curve from $4 \mathrm{mg} / \mathrm{ml}$ tubulin, $10.10 \mathrm{sec}$ after mixing with $20 \mathrm{mM}$ spermine (Fig. 4c in the main text), significantly differed from the curve at $3.28 \mathrm{sec}$ (dominated by THP structures) (g). The $10.10 \mathrm{sec}$ curve was fitted to TCS subunit model (32 dimer) in addition to the THP models (f). The best fitted mass fraction distribution contained mainly 18 and 32 dimers. 


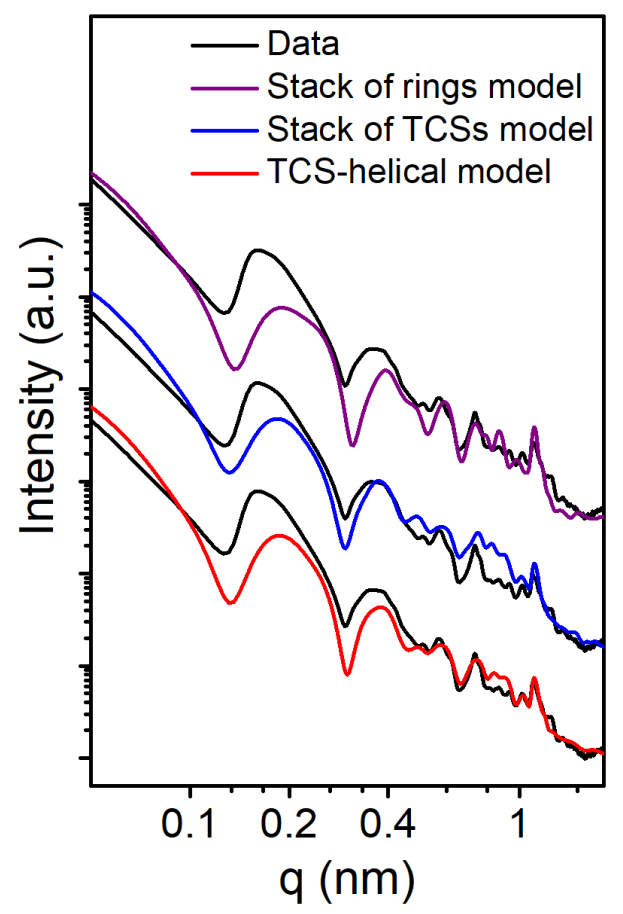

Figure 9| Fitting the scattering data to alternative models of TCSTs.

Azimuthally-averaged supernatant-subtracted synchrotron SAXS curve from $4 \mathrm{mg} / \mathrm{ml}$ tubulin with $5 \mathrm{mM}$ spermine (data, black curves) are compared with computed SAXS curves based on alternative atomic models: antiparallel tubes of subunits built from a stack of three rings with varying radii (stack of rings , purple curve), antiparallel tubes built from of a stack of TCS subunits (stack of TCS , blue curve), and antiparallel tubes built from TCS subunits, connected to each other by half a pitch protofilament (TCS-helical, red).

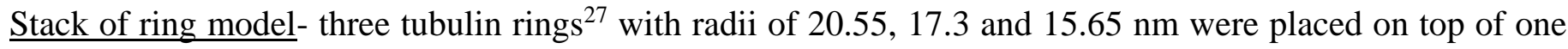
another (the largest ring was placed at the bottom and the smallest at the top) at vertical displacements of 5.72 $\mathrm{nm}$. To create a tube, we duplicated the stack of three rings in the vertical direction. The antiparallel alignment was obtained by duplicating one tube and flipping its direction by $180^{\circ}$ vertically and horizontally where the center-to-center distance between the two tubes was $46 \mathrm{~nm}$.

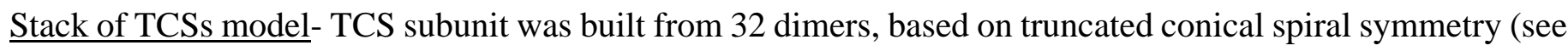
Methods) with a pitch, $p$, of $5.72 \mathrm{~nm}$, initial radius of $22.3 \mathrm{~nm}$, a final radius of $15 \mathrm{~nm}$, and 2.5 helical pitches. To create a tube, we duplicated the TCS subunit in the vertical direction. The antiparallel alignment was obtained by duplicating one tube and flipping its direction by $180^{\circ}$ vertically and horizontally where the center-to-center distance between the two tubes was $44.5 \mathrm{~nm}$.

TCS helical model- see symmetry in the Method section and parameters in the main text. 

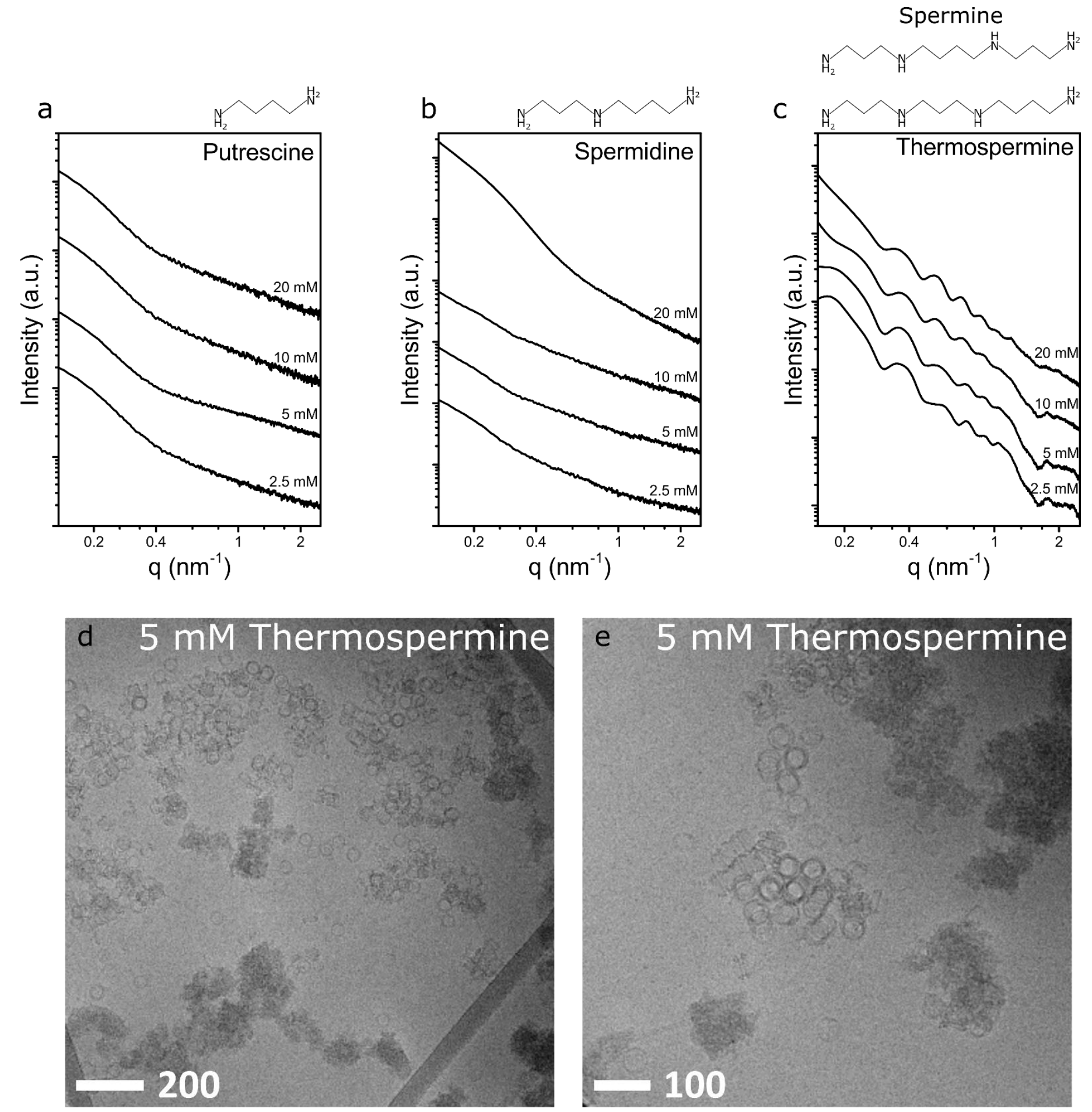

\section{Figure 10| Tubulin assembly in the presence of polyamines.}

a-c. Azimuthally-averaged supernatant-subtracted SAXS curves from $4 \mathrm{mg} / \mathrm{ml}$ tubulin incubated for $48 \mathrm{~h}$ at 9 ${ }^{\circ} \mathrm{C}$ with putrescine (a), spermidine (b) or thermospermine (c). The structure of each polyamine is shown above the figures. d, e. Cryo-TEM images of $4 \mathrm{mg} / \mathrm{ml}$ tubulin incubated for $48 \mathrm{~h}$ at $9{ }^{\circ} \mathrm{C}$ with $5 \mathrm{mM}$ thermospermine. TCS subunits and nonspecific aggregated were observed. 
a

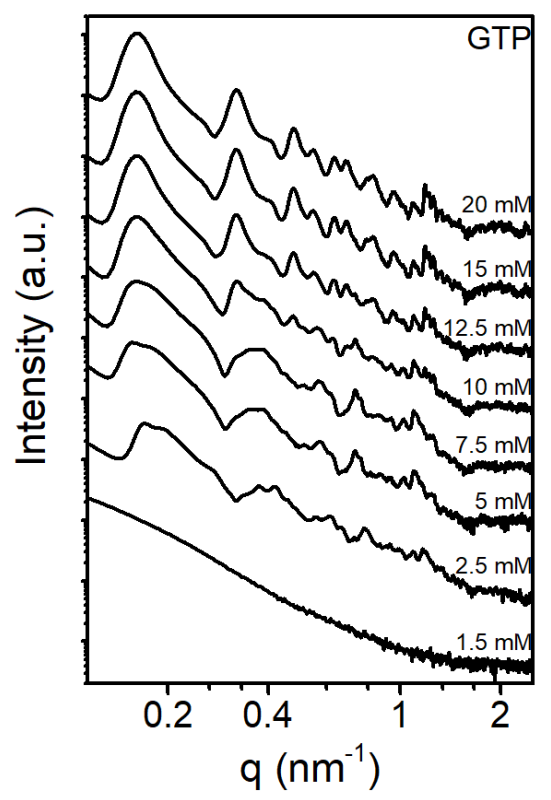

C

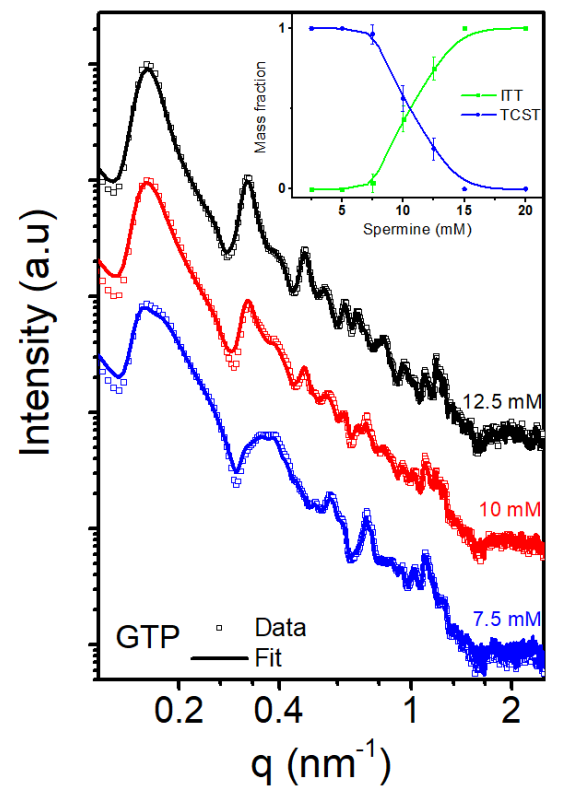

b

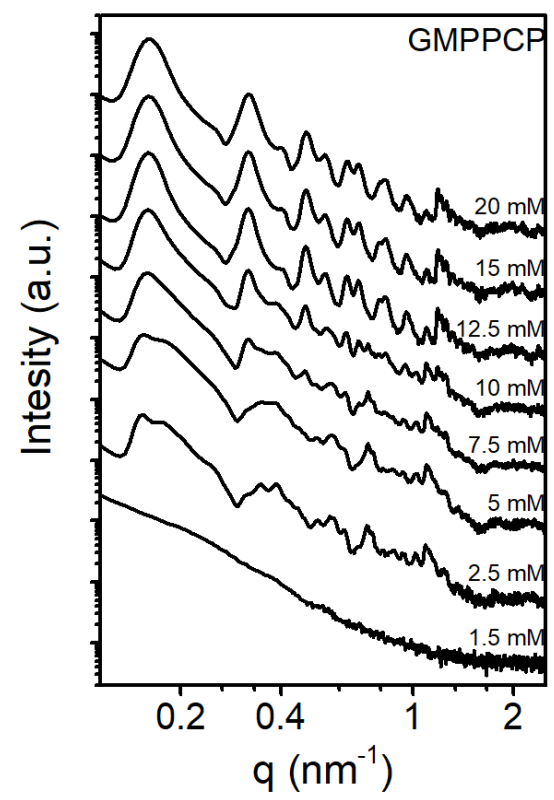

0

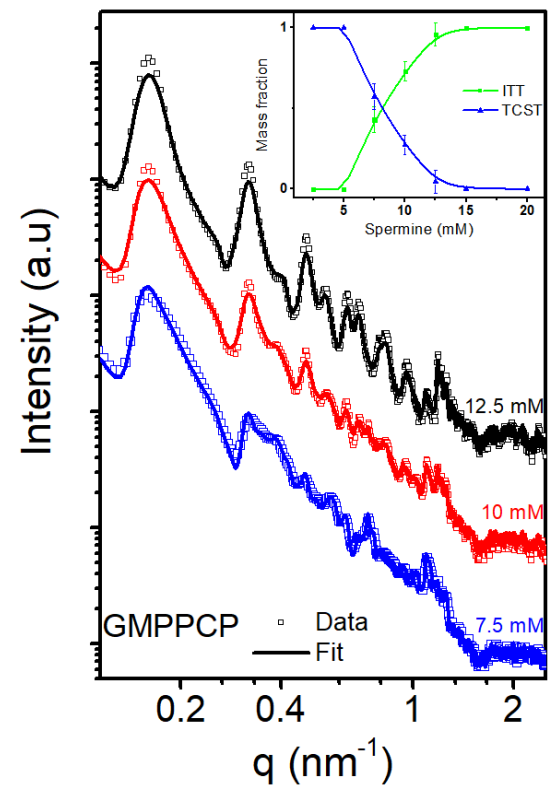

Figure 11| MT polymerized with GTP or GMPPCP formed TCSTs and ITTs in the presence of spermine.

a, b. Azimuthally-averaged supernatant-subtracted synchrotron SAXS curves of $8 \mathrm{mg} / \mathrm{ml}$ tubulin which was polymerized with $4 \mathrm{mM}$ GTP or GMPPCP $\left(45 \mathrm{~min}\right.$ at $\left.36^{\circ} \mathrm{C}\right)$. The polymerized MT was then mixed with equal volume of spermine solutions and incubated for $48 \mathrm{~h}$ at $9^{\circ} \mathrm{C}$. The final concentration of tubulin was $4 \mathrm{mg} / \mathrm{ml}$. The final concentration of GTP (a) or GMPPCP (b) was $2 \mathrm{mM}$, and the spermine concentration was between 1.5 and $20 \mathrm{mM}$, as indicated. c, d. Azimuthally-averaged supernatant-subtracted synchrotron SAXS curves (open square symbol) from $4 \mathrm{mg} / \mathrm{ml}$ GTP-tubulin (c) or GMPPCP-tubulin (d) incubated with 7.5-12.5 mM 
spermine, fitted to a linear combination (solid curves) of experimental scattering curves that mostly contained TCST or ITTs. The fit was done by a linear combination of the $5 \mathrm{mM}$ spermine signal (TCST-rich) and the $15 \mathrm{mM}$ spermine signal (ITT-rich). The insets show the mass fraction of the contributing assemblies. 
a

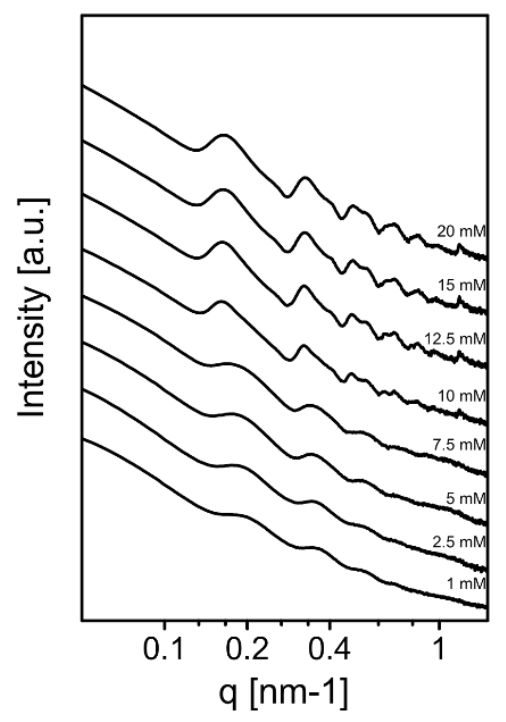

$\mathrm{C}$

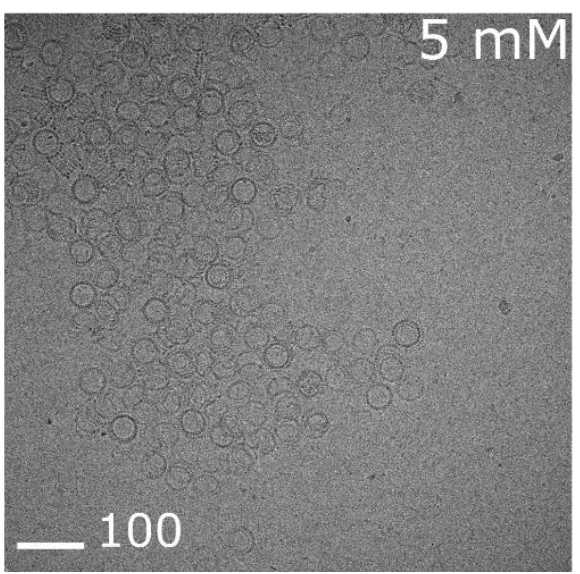

b

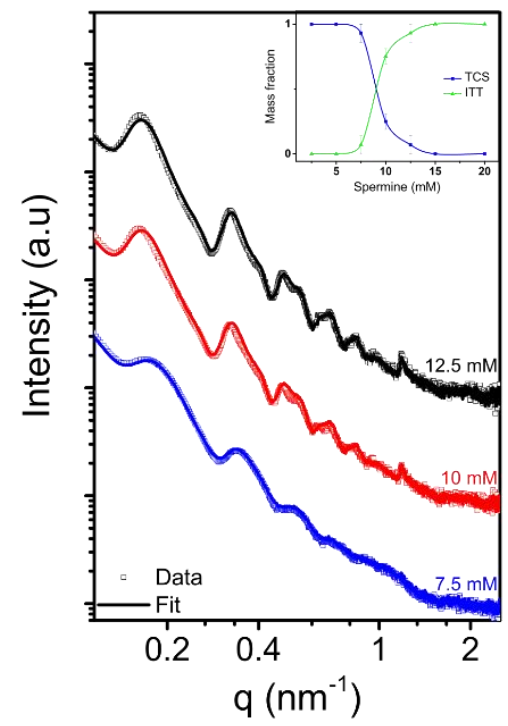

e

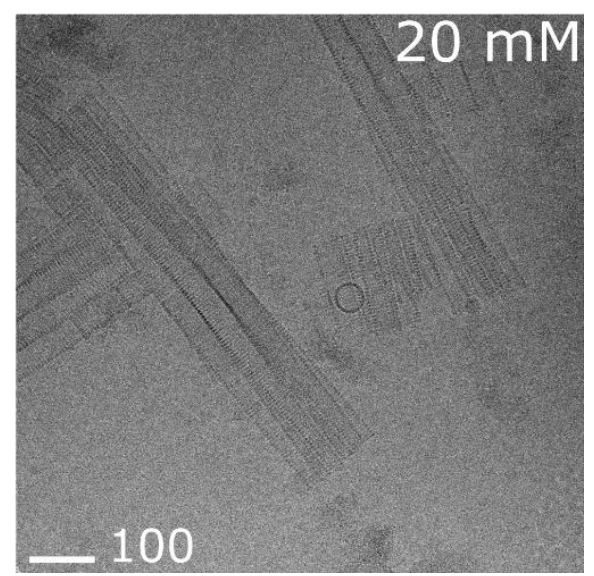

\section{Figure 12| TCSs and ITTs in the presence of MAPs.}

a. Azimuthally-averaged supernatant-subtracted synchrotron SAXS curves of $4 \mathrm{mg} / \mathrm{ml}$ tubulin with MAPs, incubated with 1 - $20 \mathrm{mM}$ spermine, after incubation at $9{ }^{\circ} \mathrm{C}$ for $48 \mathrm{~h}$. b. Azimuthally-averaged supernatantsubtracted synchrotron SAXS curves (open square symbol) from $4 \mathrm{mg} / \mathrm{ml}$ tubulin with MAPs incubated with 7.5-12.5 mM spermine, fitted to a linear combination (solid curves) of experimental scattering curves that mostly contained TCS assemblies or ITTs. The fit was done by a linear combination of the $5 \mathrm{mM}$ spermine signal (TCS-rich) and the $15 \mathrm{mM}$ spermine signal (ITT-rich). The insets show the mass fraction of the contributing assemblies. c-e. Cryo-TEM images of $4 \mathrm{mg} / \mathrm{ml}$ tubulin and MAPs with different spermine concentrations. At $5 \mathrm{mM}$ spermine, THPs coexisted with individual TCS subunits (c). At $10 \mathrm{mM}$ spermine TCS subunits coexisted with ITTs (d). At $20 \mathrm{mM}$ spermine only ITT were observed (e). 